TRANSACTIONS OF THE

AMERICAN MATHEMATICAL SOCIETY

Volume 365, Number 5, May 2013, Pages 2681-2722

S 0002-9947(2012)05709-6

Article electronically published on November 20, 2012

\title{
PI-VARIETIES ASSOCIATED TO FULL QUIVERS OF REPRESENTATIONS OF ALGEBRAS
}

\author{
ALEXEI BELOV-KANEL, LOUIS H. ROWEN, AND UZI VISHNE
}

\begin{abstract}
In an earlier paper, we introduced the notion of full quivers of representations of algebras, which are more explicit than quivers of algebras. Here, we consider full quivers (as well as pseudo-quivers) as a combinatoric tool in order to describe PI-varieties of algebras. Each full quiver is naturally associated to a polynomial that encapsulates trace-like properties of the underlying algebra.
\end{abstract}

\section{INTRODUCTION}

Recall [6, pp. 28ff.] that an algebra $A$ over an integral domain $C$ is representable if it can be embedded as a $C$-subalgebra of $\mathrm{M}_{n}(K)$ for a suitable field $K \supset C$ (which can be much larger than $C$ ). In [8] we considered the full quiver of a linear representation of an associative algebra, which is somewhat more explicit than the "classical" quiver of an algebra, and which enables one to study the structure of Zariski closed algebras, a useful generalization of finite dimensional algebras when the base field is finite. We determined properties of full quivers by means of a close examination of the structure of Zariski closed algebras. Then the full quiver was used to study the interactions between the radical and the semisimple component of Zariski closed algebras. In [8, Theorem 6.8] we saw that the Zariski closure of a representable affine PI-algebra over an infinite field has a representation whose full quiver has only proportional gluing.

Since every variety contains a relatively free algebra, we can study the PIs in terms of relatively free algebras of Zariski closed algebras. In this paper, after starting with a more thorough description of some of these results (specifically Corollary [3.8, which says that every relatively free algebra is a subdirect product of relatively free algebras having "enhanced canonical" full quivers), we consider the opposite point of view: We use the full quiver and "pseudo-quiver" to describe directly certain nonidentities and identities of a representable algebra A. Although not every affine PI-algebra is representable, every relatively free affine PI-algebra $A$ turns out to be representable, by a theorem of Kemer [16], so the theory of this paper enables us to study T-ideals of PI-algebras over an arbitrary field.

In Theorem 2.11 we show that over an infinite field, any pseudo-quiver can be reduced to a finite set of full quivers, so the concept of a pseudo-quiver is needed only over finite base fields.

Received by the editors May 12, 2011 and, in revised form, August 31, 2011 and September $20,2011$.

2010 Mathematics Subject Classification. Primary 16R10; Secondary 16R30.

This research was supported by the Israel Science Foundation (grant No. 1178/06).

(C)2012 American Mathematical Society Reverts to public domain 28 years from publication 
The main motivation for this paper is to obtain a natural nonzero T-ideal of $A$ (obtained from the nonidentity) that has special properties to be described below, thereby providing an inductive step for arguments in PI-theory, including the inductive step for Belov's proof of Specht's conjecture for affine algebras over finite fields; cf. [10]. At the same time, the full quiver also provides interesting examples of identities, which we explore but do not categorize here in a systematic way.

There already exists a very well-researched representation theory of f.d. algebras, but it is not fine enough to distinguish PIs, since the Morita equivalent algebras $\mathrm{M}_{n}(F)$ and $\mathrm{M}_{m}(F)$ satisfy different PIs for $m \neq n$. In this paper, we study how one can pass back from full quivers and varieties of algebras. We start with the full quiver (and pseudo-quiver) as cycle-free directed graphs endowed with various additional information encoded in "gluing" of vertices and of edges. Then we use the full quiver (especially when it is a single path) of an algebra to obtain certain PIs, and also to build PI-algebras from full quivers. We obtain nonidentities for the algebra $A$ of a path, having special combinatoric properties that provide algebraic insight about the corresponding relatively free PI-algebras. This is accomplished by the construction of trace-absorbing nonidentities, which by definition are closed under multiplication by traces.

To achieve this task, first we need the following "First Canonization Theorem":

Theorem ([8, Theorem 6.11]). Any relatively free affine PI-algebra A has a representation for whose full quiver all gluing is Frobenius proportional.

In this paper, we continue to focus on algebras over fields, and rely on the celebrated theorem of Shirshov [6, Chapter 2].

Remark 1.1. For some of our arguments we need to recall that Shirshov's Theorem permits us to adjoin finitely many characteristic coefficients to a given affine $C$ algebra $A$ to obtain an algebra $\hat{A}$ finite as a module over the commutative algebra $\hat{C}$ obtained by adjoining the same characteristic coefficients to $C$.

When we are given a representation $\rho: A \rightarrow \mathrm{M}_{n}(C)$, we stipulate that the generators of $\hat{C}$ include every characteristic coefficient of products of a given finite set of generators of $\rho(A)$ (viewed as a matrix in $\mathrm{M}_{n}(C)$ ).

We start by proving the following "Canonization Theorems" about reducing full quivers to a better form:

- Second Canonization Theorem 3.7 Any basic full quiver (resp. pseudoquiver) of a relatively free PI-algebra can be modified (via a change of base) to an "enhanced canonical full quiver (resp. pseudo-quiver)".

- Corollary 3.8 Any relatively free algebra is a subdirect product of algebras with representations whose full quivers are enhanced canonical.

The next result describes what happens when one mods out a "nice" T-ideal; cf. Definition 3.9

- Third Canonization Theorem 3.12 Suppose A is a relatively free PI-algebra over $C$ with pseudo-quiver $\Gamma$, and $\mathcal{I} \neq 0$ is a $C$-closed $T$-ideal of $A$. Then $A^{\prime}=A / \mathcal{I}$ is obtained by means of the following elementary operations: gluing, new linear dependences on the vertices, and new relations on the base ring.

Our trace-absorbing nonidentity then is obtained in Theorem 5.14 , by means of a "hiking procedure" which forces substitutions of a polynomial into the radical. The 
outcome is that two definitions of trace actions (one in terms of matrix computations and one in terms of polynomial evaluations) coincide on the T-ideal that is generated by this polynomial, which thus is a common ideal of $A$ and the algebra $\hat{A}$ obtained by adjoining traces to $A$, which by Shirshov's Theorem [6, Chapter 2] is Noetherian, in fact a finite module over a commutative affine algebra when $A$ is affine. This yields the next theorem, which is the main result of our paper. Recall from [8] that a maximal subpath of a full quiver or pseudo-quiver is called a branch; the length of a branch $\mathcal{B}$ is its number of arrows, which equals its number of vertices, minus 1 .

Theorem (Trace Adjunction Theorem 5.16). For any branch of a basic full quiver of a relatively free algebra $A$, there is a (naturally described) polynomial whose $T$ ideal is also an ideal of the algebra $\hat{A}$ obtained by taking $A$ together with the traces adjoined.

In characteristic 0 all characteristic coefficients can be obtained from the trace, by means of Newton's formulas. But in characteristic $p$ one has a slightly more complicated version, using all characteristic coefficients instead of just the trace, as indicated in [10. We present the trace version here, since it is enough for our applications in this paper and in [9, although we need the characteristic $p$ version in $[10$.

The Trace Adjunction Theorem provides a powerful inductive tool which will be seen in a later paper to be a key feature in the solution of Specht's problem over finite fields, but meanwhile yields some immediate applications to PI-theory, which include:

- A straightforward proof of the rationality of the Hilbert series of a relatively free PI-algebra.

- Construction of a parametric PI which is not defined over the base field (Example 7.2).

The proof of the rationality of the Hilbert series of a relatively free PI-algebra is described briefly in (elaborated upon in [9]) since it is a clear application indicative of the power of the inductive procedure. The next application is given in its entirety in this paper (Example 7.2), since it quickly illustrates the use of full quivers in constructing identities and is needed in our subsequent exposition on Specht's problem. Two other applications are provided in 9 .

In $\$ 8$ we switch our perspective, and examine other interesting PIs that arise mainly from gluing.

\section{BACKGROUND}

Remark 2.1. Any representable algebra $A \subseteq \mathrm{M}_{n}(K)$ has its Wedderburn block form described in detail in [7] and [8, Definition 3.10], which is the keystone of [8]. This Wedderburn block form induces an action of $A$ on $\mathrm{M}_{n}(K)$, by which we view each element $a \in A$ as a linear operator $\ell_{a}$ on $V$ via left multiplication. (Likewise, we also have a right action via right multiplication.) In the sequel, we usually consider the algebra $A$ in this context. 
2.1. Pseudo-quivers. Rather than restrict our view of a full quiver in terms of a given representation of an algebra, we would like a more self-contained graphtheoretic concept, whose properties are derived from those of the original algebra.

For example, for $A$ Zariski closed, when we write $A / \operatorname{Rad}(A)=\bigoplus R_{i}$ (via its Wedderburn decomposition), where $R_{i} \cong \mathrm{M}_{n_{i}}\left(F_{i}\right)$, for subfields $F_{i}$ of $K$, each set of glued vertices of the full quiver corresponds to the idempotent $e_{i}$ which is the unit element of $R_{i}$. In particular, all identifications among vertices of a full quiver are consequences of identical or Frobenius gluing.

For further reference, we also bring in the slightly more general notion of pseudoquiver, to enable linear changes of basis in the representation. When passing to the pseudo-quiver, we often sacrifice the property described in the previous paragraph, as indicated in Remark 2.7 below.

We write $\mathbb{F}_{q}$ for the field of $q$ elements.

Definition 2.2. A pseudo-quiver is a directed graph $\Gamma$, without double edges and without cycles, having the following information attached to the vertices and edges:

- As with full quivers, the vertices are ordered, say from $\mathbf{1}$ to $\mathbf{k}$, and an edge can only take a vertex to a vertex of higher order; in particular, the pseudoquiver has no cycles and no loops. There also are identifications of vertices and of edges, called gluing. Identification of vertices is called diagonal gluing, whereas identification of edges is called off-diagonal gluing.

- Each vertex is labelled with a roman numeral (I, II, etc.); glued vertices are labelled with the same roman numeral. A vertex can be either filled or empty. Empty vertices are denoted as o. When there is no gluing among the vertices, each filled vertex is labelled as $\bullet$, rather than a roman numeral.

When the base field $F$ is finite, i.e., $F=\mathbb{F}_{q}$ for some $q$, superscripts $\ell$ for the roman numerals indicate the Frobenius twist, which will correspond to a Frobenius endomorphism $\phi$ of the underlying algebra. The upper label $I^{(\ell)}$ indicates that the vertex is obtained from another vertex, $I$, by applying $\phi^{\ell}$.

The first vertex listed in a glued component is also given a pair of subscripts in the form $n_{\mathbf{i}}\left(t_{\mathbf{i}}\right)$ : the matrix degree $n_{\mathbf{i}}$ and the cardinality of the corresponding field extension of $F$ (which, when finite, is denoted as a power $q^{t_{\mathbf{i}}}$ of $q=|F|$ ). (All the other vertices in the same glued component are considered to have this same matrix degree and same cardinality.)

- As with full quivers, an edge $(\mathbf{i}, \mathbf{j})$ is called primitive if there is no $\mathbf{k}$ such that there exist edges $(\mathbf{i}, \mathbf{k})$ and $(\mathbf{k}, \mathbf{j})$. Only primitive edges are notated, unless gluing dictates us to notate a nonprimitive edge.

- Off-diagonal gluing (i.e., gluing among the edges) has several possible types, including Frobenius gluing and proportional gluing with an accompanying scaling factor. Absence of a scaling factor indicates scaling factor 1 ; such gluing is called identical gluing when there is no Frobenius twist indicated.

Remark 2.3. Each gluing of vertices in full quivers is identical or Frobenius. In contrast with full quivers, the vertices of a pseudo-quiver could carry linear relations that are not consequences of identical or Frobenius gluing. We do not provide a notation for this circumstance. 
If two primitive edges in $\Gamma$ are Frobenius glued, with respect to $q, q^{\prime}$, then their initial (resp. terminal) vertices have the same Frobenius twist.

Remark 2.4. In [8, $\S \S 5.1,5.2$, and 7.1] (and especially [8, Example 5.4]) we described a method (which we review for the reader's convenience), called compression, of modifying a full quiver to make it more concise, and which involves changing the base field into a commutative affine algebra generated by elements $\xi_{i}$ involving new relations that provide nilpotent elements which could be viewed as "infinitesimals". Namely, gluing within a branch could lead to the following sort of situation:

$$
B_{i}=\left\{\left(\begin{array}{ccccc}
\alpha & \beta & \gamma & \ldots & \cdots \\
0 & \alpha & \beta & \ddots & \vdots \\
0 & 0 & \ddots & \ddots & \gamma \\
0 & 0 & 0 & \alpha & \beta \\
0 & 0 & 0 & 0 & \alpha
\end{array}\right): \alpha, \beta, \gamma \in K\right\}
$$

This algebra is isomorphic to $F\left[\xi_{i}\right] /\left\langle\xi_{i}^{\ell}\right\rangle$, where $\ell$ is the size of this matrix block $B_{i}$, and we replace the entire block $B_{i}$ by this single vertex.

Definition 2.5. A relation is called gluing up to infinitesimals if it has the form:

$$
\left\langle\xi_{i}^{q^{t}}-\xi_{j}\right\rangle^{k}=0
$$

for suitable $i, j$ and a suitable $k$.

Note that when $i=j$ this merely means that we are adjoining an algebraic element to the base field to obtain a commutative algebra which may have nilpotent elements.

The trickiest aspect of the subject is the interaction of identities caused by gluing between branches. Here are some extreme cases.

Definition 2.6. Gluing between branches is called degenerate if each edge of one branch is glued to the corresponding edge of the other branch, in the same order.

Gluing between branches is permuted if the edges (including labeling) are permuted from the first branch to the second branch.

A permuted branch of a given branch $\mathcal{B}$ is a branch with permuted gluing to $\mathcal{B}$.

We say that a vertex $r$ precedes $s$ (written $r \prec s$ ) if there is a path from $r$ to $s$. This relation is antisymmetric since $\Gamma$ has no cycles.

In $[8$, since we were concentrating on the algebra representations, we used the terminology "arrow" instead of "edge". Here, arrow denotes the edge together with its two terminal vertices. Two vertices joined by a primitive arrow are called consecutive.

Remark 2.7. Any full quiver of a representation of an algebra $A$ gives rise to a pseudo-quiver. Starting with the $k$ vertices $v_{1}, \ldots, v_{k}$ corresponding to central idempotents of the blocks, we proceed as in Remark 2.1. We identify the entries of the respective blocks according to gluing (with identical gluing identifying entries and with Frobenius gluing corresponding to the Frobenius automorphism). For any two idempotents $e_{\mathbf{i}}, e_{\mathbf{j}}$ we choose a base of $e_{\mathbf{i}} A e_{\mathbf{j}}$ for the arrows between $\mathbf{i}$ and $\mathbf{j}$. 
Then by definition, any two consecutive vertices have only a single arrow joining them, although new linear dependence may arise among vertices corresponding to the same idempotents.

A morphism $\psi: \Gamma_{1} \rightarrow \Gamma_{2}$ of full quivers (or of pseudo-quivers) is a morphism of directed graphs (i.e., $\psi((\mathbf{i}, \mathbf{j}))=(\psi(\mathbf{i}), \psi(\mathbf{j}))$ for each edge $(\mathbf{i}, \mathbf{j}))$, preserving gluing, such that if $n_{\mathbf{i}}\left(t_{\mathbf{i}}\right)$ is the subscript for the vertex $\mathbf{i}$, then $\mathrm{M}_{n_{i}}\left(\mathbb{F}_{q^{t_{i}}}\right)$ embeds into $\mathrm{M}_{n_{\psi(\mathbf{i})}}\left(\mathbb{F}_{q^{t} \psi(\mathbf{i})}\right)$, whereby the superscripts are carried with their respective vertices.

We say that $\Gamma_{1}$ is a subquiver of $\Gamma_{2}$ if there is a 1:1 morphism from $\Gamma_{1}$ to $\Gamma_{2}$. In other words, we get a subquiver passing to a subalgebra in a vertex, or by erasing vertices and the edges attached to them.

\subsection{Representations of algebras on a subquiver or sub-pseudo-quiver.}

We want to elaborate on [8, Corollary 7.2], which is an important tool in studying full quivers and pseudo-quivers.

Recalling that Remarks 2.1 and 2.7 enable us to view the elements of $A$ as operators, we can also represent $A$ as acting on a full quiver or pseudo-quiver of its representation, via composition of linear maps. More precisely, $A$ acts on $V=K^{(n)}$, which is written as a direct sum of $K$-spaces of dimension 1 , each corresponding to a vertex of $\Gamma$. (Of course, one also takes gluing into account when describing these operators.)

Definition 2.8. Given a sub-(pseudo-)quiver $\Gamma^{\prime}$ of a (pseudo-)quiver $\Gamma$ of $A$, one can define the algebra $A_{\Gamma^{\prime}}$ to be the algebra obtained by restricting the action of the elements of $A$ to $\Gamma^{\prime}$. Explicitly, given $a \in A$, define $a_{\Gamma^{\prime}}$ to be the composition of $\left.\ell_{a}\right|_{\Gamma^{\prime}}$ with the projection sending the vector space corresponding to the sum of all blocks not in $\Gamma^{\prime}$ to 0 .

We say that a subgraph $\Gamma_{0}$ of $\Gamma$ is convex when the following property holds:

If the concatenation $p_{1} p_{2}$ of two paths of $\Gamma$

lies in $\Gamma_{0}$, then $p_{1}$ and $p_{2}$ both lie in $\Gamma_{0}$.

Proposition 2.9. If $\Gamma^{\prime}$ is a convex subquiver (resp. sub-pseudo-quiver) of a full quiver (resp. pseudo-quiver) $\Gamma$, then the natural map $a \mapsto a_{\Gamma^{\prime}}$ defines an algebra surjection.

Proof. By definition, this is a vector space projection, so we need to prove that it preserves multiplication. But the only way for multiplication not to be preserved is for the concatenation of two paths not in $\Gamma^{\prime}$ to yield a path between two vertices of $\Gamma^{\prime}$, thereby contradicting the assumption that $\Gamma^{\prime}$ is convex.

We call a pseudo-quiver 2-reduced if it has no relations of the form $\gamma_{1} v_{1}+\gamma_{2} v_{2}=$ 0 for $\gamma_{1}$ invertible.

Lemma 2.10. Any algebra has a representation with a 2-reduced pseudo-quiver.

Proof. The relation $\gamma_{1} v_{1}+\gamma_{2} v_{2}=0$ can be eliminated simply by solving $v_{1}=-\frac{\gamma_{2}}{\gamma_{1}} v_{2}$ and coalescing the vertices (since the arrows are viewed as linear operators).

We always assume in the sequel that our pseudo-quivers are 2-reduced. Although this process cannot be done for longer relations, we have the following trick. 
Lemma 2.11. If the base field $F$ is infinite, then for any pseudo-quiver $\Gamma$, and any linear relation $\sum_{k=1}^{t} \alpha_{i_{k}} v_{i_{k}}=0$ among the vertices, its algebra $A$ is a finite subdirect product of algebras having the same pseudo-quiver as $\Gamma$ (perhaps with some arrows erased) except that this relation has been eliminated.

Proof. We need to resolve linear relations among the vertices. Suppose $\sum_{k=1}^{t} \alpha_{i_{k}} v_{i_{k}}$ $=0$. For convenience we assume that $i_{1}=1$. For each $1<k<t$ we consider the $v_{i_{k}}$ obtained by erasing all arrows leading to or from $v_{i_{k^{\prime}}}, k^{\prime} \neq k$. This sub-pseudoquiver is convex, so by Proposition 2.9 its algebra is a homomorphic image of $A$.

Theorem 2.12. If the base field $F$ is infinite, then for any pseudo-quiver $\Gamma$, its algebra $A$ is a subdirect product of algebras whose reduced pseudo-quivers are images of $\Gamma$.

Proof. Since $F$ is infinite, all Frobenius gluing is identical, so we are done by repeated applications of Lemma 2.11.

\subsection{The dual full quiver.}

Definition 2.13. The dual quiver $\Gamma^{\mathrm{op}}$ of a full quiver (resp. pseudo-quiver) $\Gamma$ is obtained by reversing the direction of the arrows of $\Gamma$.

Proposition 2.14. If $\Gamma$ is the full quiver (resp. pseudo-quiver) of an algebra $A \subseteq$ $\mathrm{M}_{n}(K)$, then $\Gamma^{\mathrm{op}}$ is the full quiver (resp. pseudo-quiver) of the transpose of $A$.

Proof. The representations of $\Gamma^{\mathrm{op}}$ correspond to the transposes of the representations of $A$; switching indices $i$ and $n+1-i$ for each $1 \leq i \leq n$ reverses the arrows.

2.4. Varieties of PI-algebras. Algebras are called PI-equivalent if they satisfy the same polynomial identities (PIs). One of the major ongoing problems of PItheory is to classify PI-algebras up to PI-equivalence, or, equivalently, classifying varieties according to the PIs defining them. Let us call this question the "PI Classification Problem". From this standpoint, the celebrated theorem of Kemer [16] (answering Specht's problem) can be formulated as saying that for any algebra of characteristic 0 , its set of PIs is a consequence of a finite set of PIs. Later, Kemer [18] extended his result to algebras over arbitrary infinite fields.

In his proof, as described in 6, Chapter 4], Kemer shows that any affine PIalgebra over an infinite field is PI-equivalent to a finite dimensional (f.d.) algebra, and proceeds to describe their varieties in terms of identities of minimal "Kemer index". This could be viewed as a first step in understanding the PIs of f.d. algebras, the objective being to differentiate among different varieties of PIs by means of certain distinguished identities and nonidentities.

The set $\operatorname{id}(A)$ of polynomial identities of an algebra $A$ is well known to be a T-ideal of the free algebra built from a countable set of indeterminates over the base field. More generally, given a polynomial $f$, we define $f(A)^{+}$to be the linear subspace of $A$ spanned by $f(A)$. Thus, $f(A)^{+}=0$ iff $f \in \operatorname{id}(A)$.

We call an algebra $A$ representation-irreducible if for any two nonzero ideals $I_{1}, I_{2}$ such that $A / I_{1}$ and $A / I_{2}$ are representable, their intersection $I_{1} \cap I_{2}$ is nonzero. If $I_{1}, I_{2} \subseteq A$ with $I_{1} \cap I_{2}=0$, then $A \hookrightarrow A / I_{1} \times A / I_{2}$, implying that $\operatorname{id}(A)=$ $\operatorname{id}\left(A / I_{1}\right) \cap \operatorname{id}\left(A / I_{2}\right)$. Thus, the PI classification problem can be reduced to the case of representation-irreducible algebras. 
Given a T-ideal $\mathcal{I}$ of the free algebra $F\{x\}$, we can form the relatively free algebra $F\{x\} / \mathcal{I}$, which is free in the class of all PI-algebras $A$ for which $\operatorname{id}(A) \subseteq \mathcal{I}$. Using this correspondence, it is enough to classify relatively free algebras in terms of the full quivers of their representations. We investigate relatively free PI-algebras in terms of their generic elements, as constructed in [7, Construction 7.14] and studied in [7, Theorem 7.15]. (A generic element of a finite dimensional algebra having base $\left\{b_{1}, \ldots, b_{n}\right\}$ over an infinite field is just an element of the form $\sum \xi_{i} b_{i}$, where the $\xi_{i}$ are indeterminates, but the situation for algebras over a finite field becomes considerably more intricate.)

As with most combinatorial studies of polynomial identities, we rely heavily on the Capelli polynomial

$$
c_{k}\left(x_{1}, \ldots, x_{k} ; y_{1}, \ldots, y_{k}\right)=\sum_{\pi \in S_{k}} \operatorname{sgn}(\pi) x_{\pi(1)} y_{1} \cdots x_{\pi(k)} y_{k}
$$

of degree $2 k$ (cf. [6]). Any $C$-subalgebra of $\mathrm{M}_{n}(K)$ satisfies the identities $c_{k}$ for all $k>n^{2}$.

Definition 2.15. We denote by $h_{n}$ the $n^{2}$-alternating central polynomial on $n \times n$ matrices [6, p. 25], which we recall is

$$
g_{n}\left(c_{n^{2}}\left(x_{1}, \ldots, x_{n^{2}}, y_{1}, \ldots, y_{n^{2}}\right), x_{n^{2}+1}, x_{n^{2}+d-1}\right),
$$

where $g_{n}$ is a multilinear central polynomial for $n \times n$ matrices. Also, we define formally $h_{0}=1$.

When appropriate, we write $h_{n}(x)$ to emphasize that $h_{n}$ is evaluated on indeterminates $x_{1}, x_{2}, \ldots$

Remark 2.16. $h_{n}$ is a crucial polynomial for our goal, since it is always central for $\mathrm{M}_{n}(C)$ regardless of the commutative base ring $C$.

Furthermore, 6, Theorem J, p. 27] shows for any $t$-alternating polynomial $f$ where $t=n^{2}$, that $f(A)^{+}$is closed under multiplication by characteristic coefficients of linear transformations. More precisely, [6, Theorem J, Equation (1.18)] says

$$
\alpha_{k} f\left(x_{1}, \ldots, x_{t}, y_{1}, \ldots, y_{m}\right)=\sum f\left(z^{k_{1}} x_{1}, \ldots, z^{k_{t}} x_{t}, y_{1}, \ldots, y_{m}\right)
$$

summed over the $\left(\begin{array}{l}t \\ k\end{array}\right)$ vectors $\left(k_{1}, \ldots, k_{n}\right)$ for which each $k_{i} \in\{0,1\}$ and $\sum k_{i}=k$, where $\alpha_{k}$ is the coefficient of $\lambda^{t-k}$ in the characteristic polynomial of the transformation $T$ given by left multiplication by $z$ (viewed as a transformation of $t$-dimensional space, and thus as a $t \times t$ matrix). From this point of view, $\alpha_{k}$ could be viewed as the quotient of two polynomials. Note that $\alpha_{1}$ is $-n \operatorname{tr}(z)$. This is the key to "trace absorption", discussed in Definition 5.3.

2.4.1. Reductions to certain kinds of polynomials. We want to focus on the kinds of polynomials that arise naturally in the theory of pseudo-quivers.

Lemma 2.17. Suppose that $W \supset F$ is a commutative $F$-algebra.

(1) If $F$ is infinite, then $\operatorname{id}\left(\mathrm{M}_{n}(W)\right)=\operatorname{id}\left(\mathrm{M}_{n}(F)\right)$.

(2) If $F$ is finite, then $\operatorname{id}\left(\mathrm{M}_{n}(W)\right) \subsetneq \operatorname{id}\left(\mathrm{M}_{n}(F)\right)$.

Proof. (1) Any algebra over an infinite field satisfies the same PIs as its tensor extension by any commutative algebra.

(2) If $h_{n}(x)$ is as in Definition 2.15, then $h_{n}\left(\mathrm{M}_{n}(F)\right)$ contains some value in $W \backslash F$, implying $\prod_{a \in F}\left(h_{n}(x)-a\right)$ is an identity of $\mathrm{M}_{n}(F)$ but not of $\mathrm{M}_{n}(W)$. 
Also note, for $|F|=q$, that $h_{n}^{q}-h_{n}$ is an identity of $\mathrm{M}_{n}(F)$, which will only be an identity of $\mathrm{M}_{n}(W)$ when $x^{q}=x$ for all $x$ in $W$.

Lemma 2.18. If $W$ is not commutative, then

$$
\operatorname{id}\left(\mathrm{M}_{n}(W)\right) \subsetneq \operatorname{id}\left(\mathrm{M}_{n}(F)\right) .
$$

Proof. The Capelli polynomial $c_{n^{2}+1}\left(x_{1}, \ldots, x_{n^{2}+1} ; y_{1}, \ldots, y_{n^{2}+1}\right)$ is an identity of matrices over any commutative ring, but does not vanish in $\mathrm{M}_{n}(W)$ for the substitutions

$$
\begin{gathered}
x_{1}=w_{1} e_{11}, y_{1}=e_{11}, x_{2}=e_{12}, y_{2}=e_{21}, \ldots, \\
y_{n^{2}}=e_{n, 1}, x_{n^{2}+1}=w_{2} e_{11}, y_{n^{2}+1}=e_{11}
\end{gathered}
$$

for noncommuting $w_{1}, w_{2} \in W$. Indeed, the only nonzero substitutions are when $x_{1}$ and $x_{n^{2}+1}$ appear in their original positions or are interchanged, yielding the value $\left[w_{1}, w_{2}\right] e_{11}$.

(An alternative proof could be had by taking $\left[h_{n}(x), h_{n}(y)\right]$ in different sets of indeterminates.)

Definition 2.19. A polynomial is blended if each indeterminate appearing nontrivially in the polynomial appears in each of its monomials.

Remark 2.20. It is shown in 22] by specializing extraneous elements of nonblended polynomials to 0 , that each PI is a sum of blended PIs. Thus, any T-ideal is generated by blended polynomials, and we only consider blended polynomials.

Next, we recall the usual linearization procedure, in which we define

$$
\Delta_{i}(f)=f\left(\ldots, x_{i}+x_{i}^{\prime}, \ldots\right)-f\left(\ldots, x_{i}, \ldots\right)-f\left(\ldots, x_{i}^{\prime}, \ldots\right) .
$$

We call the effect of applying this linearization procedure several times, a partial linearization of $f$.

Note that if $f$ is blended, then so is $\Delta_{i}(f)$ for each $i$. Next we recall that a polynomial $f$ is $i$-quasi-linear on $A$ if

$$
f\left(\ldots, a_{i}+a_{i}^{\prime}, \ldots\right)=f\left(\ldots, a_{i}, \ldots\right)+f\left(\ldots, a_{i}^{\prime}, \ldots\right)
$$

for all $a_{i}, a_{i}^{\prime} \in A ; f$ is quasi-linear on $A$ if $f$ is $i$-quasi-linear on $A$ for all $i$.

Lemma 2.21. For any blended nonidentity $f$, some partial linearization of $f$ is a quasi-linear (but not necessarily multilinear) nonidentity on A.

Proof. The proof is by induction on $\operatorname{deg} f$ minus the number of indeterminates occurring in $f$. Applying $\Delta_{i}$ lowers this number. On the other hand, $\Delta_{i}(f) \notin \operatorname{id}(A)$ iff $f$ is not quasi-linear on $A$, so we continue applying the linearization procedure until the next step yields an identity.

Remark 2.22. The reason we work with quasi-linear polynomials on $A$ is that when $A$ is Zariski-closed, we can write $A=S \oplus J$, where $S$ is the sum of the diagonal blocks, which is semisimple, and $J$ is the radical of $A$. Thus, one can check whether a quasi-linear polynomial $f(x)$ is a PI of $A$, merely by specializing the indeterminates $x_{i}$ to $S \cup J$.

Since PIs are obtained from quasi-linear polynomials, we focus on these, thereby enabling us to limit the kinds of substitutions of indeterminates in a given polynomial $f$. A semisimple substitution (into a Zariski closed algebra $A$ ) is a substitution into a diagonal Wedderburn block (the semisimple part of $A$ ), and a 
radical substitution is a substitution into an off-diagonal Wedderburn block (the radical part of $A$ ).

We often use different letters to designate indeterminates of a given polynomial $f$ that are intended to have different kinds of substitutions.

One can also consider scalar multiplication by $F$.

Definition 2.23. A polynomial $f\left(x_{1}, \ldots, x_{n}\right)$ is $F$-homogeneous in $x_{i}$ if there is $d \in \mathbb{N}^{+}$such that, for each $\alpha \in F$,

$$
f\left(x_{1}, \ldots, \alpha x_{i}, \ldots, x_{n}\right)=\alpha^{d} f\left(x_{1}, \ldots, x_{n}\right) ;
$$

$f\left(x_{1}, \ldots, x_{n}\right)$ is completely $F$-homogeneous if $f\left(x_{1}, \ldots, x_{n}\right)$ is $F$-homogeneous in $x_{i}$ for each $1 \leq i \leq n$.

Lemma 2.24. Every (quasi-linear) identity is the sum of completely F-homogeneous (quasi-linear) identities.

Proof. Consider the identity $f\left(x_{1}, \ldots, \alpha x_{i}, x_{n}\right)-\alpha^{d} f\left(x_{1}, \ldots, x_{n}\right)$, which enables us to rewrite $f$ as a sum of identities having fewer monomials, unless $f$ is completely $F$-homogeneous. Thus, we conclude by induction on the number of monomials of $f$.

2.5. Trace functions on matrix rings. We also will need some results about matrices over arbitrary rings (not necessarily commutative).

There are two ways of formally defining traces in matrices.

Definition 2.25. In any matrix ring $\mathrm{M}_{n}(W)$, we define

$$
\operatorname{tr}_{\text {mat }}(a)=\sum_{i, j=1}^{n} e_{i j} a e_{j i} ;
$$

we call this the matrix definition $\operatorname{tr}_{\text {mat }}(a)$ of the trace.

More generally, we define arbitrary characteristic coefficients

$$
\alpha_{t}(a):=\sum_{j=1}^{n} \sum e_{j, i_{1}} a e_{i_{1}, i_{2}} \cdots a e_{i_{t}, j},
$$

the inner sum taken over all cycles $\left(i_{1}, \ldots, i_{t}=i_{1}\right)$ of length $t$.

Thus, $\operatorname{tr}_{\text {mat }}(a)=\alpha_{1}(a)$. Usually (3) is enough for our purposes; we need arbitrary characteristic coefficients when studying matrix algebras of nonzero characteristic.

Of course, these traces commute iff $W$ is a commutative ring. This is a key issue, which we will need to address.

Remark 2.26. We can also define traces and other characteristic coefficients via polynomials, as in Remark 2.16, Namely, by equation (2), for any $n^{2}$-alternating polynomial $f(x ; y)$, we can find another polynomial $\tilde{f}(x ; y ; z)$ such that

$$
n \operatorname{tr}(a) f(x ; y)=\tilde{f}(x ; y ; a)
$$

for every $a \in A$. We denote this implicit definition of the trace of $a \operatorname{as} \operatorname{tr}_{\text {pol }}(a)$.

We will want to identify these two notions of traces. Clearly $\operatorname{tr}_{\text {mat }}(a)=\operatorname{tr}_{\text {pol }}(a)$ in $\mathrm{M}_{n}(C)$ for $C$ commutative. Consequently, we have:

Proposition 2.27. $n f(x ; y) \operatorname{tr}_{\text {mat }}(a)-\tilde{f}(x ; y ; a) \in\langle[W, W]\rangle$ when evaluated in $\mathrm{M}_{n}(W)$, for any algebra $W$. 
Remark 2.28. In order to free ourselves from an assumption on the characteristic, we could view the generic matrix $z$ as a left operator on $K^{(n)}$, and apply the more general result of Zubrilin [6, Corollary 2.45, Theorem 4.82, and Corollary 4.83] instead of [6. Theorem J, p. 27] to pass directly to the characteristic polynomial of degree $n$ and define all of the characteristic coefficients in terms of polynomials. In this case, we need to utilize what is called an RZ-polynomial in [6. Definition 4.78]. Fortunately, the Capelli polynomial $c_{n^{2}}$ is an RZ-polynomial for $n \times n$ matrices, so we often replace a given polynomial $f\left(x_{1}, x_{2}, \ldots\right)$ by $f\left(c_{n^{2}}(y) x_{1} c_{n^{2}}(z), x_{2}, \ldots\right)$ (i.e., taking new indeterminates for the Capelli polynomials).

This issue can be sidestepped in this paper, in view of the trick used in Lemma 5.1 However, it cannot be avoided in the continuation, in [10], which requires an extension of Zubrilin's theory to characteristic $p$.

2.6. The algebra of a full quiver. Having seen in [8, Definition 3.3] how a full quiver arises from a representation of an algebra, we also want to proceed in the opposite direction. We need to define a correspondence from full quivers to algebras, such that glued vertices go to matrix blocks with identifications, arrows go to linear transformations given by radical elements, and Frobenius gluing becomes Frobenius relations.

Assume that the base field $F$ has order $q$ (where $q$ could be infinity). Recall, when $q<\infty$, that $q$ is a power of $p=\operatorname{char}(F)<\infty$, and the Frobenius map $a \mapsto a^{q}$ is an $F$-algebra endomorphism. We turn to the connection between full quivers and algebras.

Definition 2.29. Fix an $F$-algebra $C$. (Usually $C=F$, but sometimes a commutative affine $F$-algebra. For example, when considering relatively free algebras, it is convenient to have $C$ contain enough commutative indeterminates to permit us to define generic matrices.) Any full quiver $\Gamma$ gives rise to an algebra $A=\mathcal{A}(\Gamma)$ as follows:

- Let $n$ be the sum of the matrix degrees of all of the vertices of $\Gamma$. Let $\bar{C}=C \otimes_{F} \bar{F}$, where $\bar{F}$ is the algebraic closure of $F$. Our algebra is to be represented inside $\mathrm{M}_{n}(\bar{C})$.

The vertex labelled by $n_{\mathbf{i}}\left(t_{\mathbf{i}}\right)$ corresponds to a matrix block $\mathrm{M}_{n_{\mathbf{i}}}\left(C_{\mathbf{i}}\right)$, where $C_{\mathbf{i}} \subset \bar{C}$ is the tensor product $C \otimes_{F} F_{\mathbf{i}}$ of $C$ with the field $F_{\mathbf{i}}$ of $q_{\mathbf{i}}:=q^{t_{\mathbf{i}}}$ elements. (When $F$ is infinite, then we just take $F_{\mathbf{i}}=F$.) We call $\mathrm{M}_{n_{\mathbf{i}}}\left(C_{\mathbf{i}}\right.$ ) the block corresponding to this vertex, and these blocks are embedded naturally into $\mathrm{M}_{n}(\bar{C})$ along the diagonal. Taking the identity element $e_{\mathbf{i}}$ of each given block, we have the Peirce decomposition $\bigoplus e_{\mathbf{i}} \mathrm{M}_{n}(\bar{C}) e_{\mathbf{j}}$ of $\mathrm{M}_{n}(\bar{C})$; our algebra $A$ is represented inside the upper block triangular part $\bigoplus_{\mathbf{i} \leq \mathbf{j}} e_{\mathbf{i}} \mathrm{M}_{n}(\bar{C}) e_{\mathbf{j}}$ of $\mathrm{M}_{n}(\bar{C})$.

- The arrow from the $\mathbf{i}$ to $\mathbf{j}$ vertices corresponds to the block $e_{\mathbf{i}} \mathrm{M}_{n}(\bar{C}) e_{\mathbf{j}}$ above the diagonal. We call these the off-diagonal blocks (which necessarily belong to the radical).

- Various relations, called quiver relations, are defined in terms of the gluing in the full quiver:

Identical gluing of vertices indicates that the respective entries of the glued blocks are equal. The Frobenius twist $\ell$ (for $q<\infty$ ) for two glued diagonal blocks indicates that a given entry of the second block is obtained by raising the corresponding entry of the first block to the $q^{\ell}$ power. 
Remark 2.30. As a bookkeeping device, multiply each entry in the $\mathbf{i}$-diagonal block by a commuting indeterminate $\lambda_{\mathbf{i}}$. The finiteness conditions and gluing relations then are described in terms of relations among the $\lambda_{\mathbf{i}}$ (for example $\lambda_{\mathbf{i}}^{q_{\mathbf{i}}}-\lambda_{\mathbf{i}}$ ). Likewise, the same indeterminate is assigned to glued blocks, with the appropriate power according to Frobenius gluing. We write $\overline{F[\Lambda]}$ to denote the polynomial algebra $F\left[\lambda_{1}, \lambda_{2}, \ldots\right]$ modulo these relations.

Again, identical gluing indicates that the respective entries of these off-diagonal blocks are equal, and similarly one can apply the Frobenius twist to each offdiagonal block. Proportional gluing for off-diagonal blocks is given by applying the scaling factor to each of the entries.

Thus, the vertices of the full quiver yield diagonal blocks corresponding to matrix algebras, whereas the edges yield blocks above the diagonal, and the quiver relations are obtained by identical gluing and proportional gluing (which could be Frobenius gluing when the base field is finite).

We define id $(\Gamma)$ to be id $(\mathcal{A}(\Gamma))$, taking $C=F\left[\lambda_{\mathbf{i}}: \lambda_{\mathbf{i}}^{q_{\mathbf{i}}}=\lambda_{\mathbf{i}}\right]$.

Remark 2.31. $\Gamma(\mathcal{A}(\Gamma))=\Gamma$, since we recover the full quiver by reversing the procedure.

In the other direction, we would also like to say that if $\Gamma$ is the full quiver of a relatively free algebra $A=F\{X\} / I$, then $I=\operatorname{id}(\mathcal{A}(\Gamma))$. This requires heavier machinery, and is given in Lemma 3.10 below.

Recall that a (not necessarily commutative) algebra $W$ is called local if $W / J$ is a (skew) field, where $J$ is the Jacobson radical of $W$.

Lemma 2.32. Suppose $\Gamma$ has the full quiver

$$
I_{n(t)} \longrightarrow I \longrightarrow \ldots \longrightarrow I \text {. }
$$

Then the corresponding algebra $A=\mathrm{M}_{n}(W)$, where $W$ is a local algebra over $F$.

Proof. We rely on some standard facts about idempotent-lifting, given in 23 . By hypothesis, $A$ has a set of $n \times n$ matrix units, which implies by [23, Proposition 1.1.3] that $A \cong \mathrm{M}_{n}(W)$ for some algebra $W$, which cannot have any idempotents since all the other vertices are glued. Furthermore, the full quiver shows that the semisimple part is $\mathrm{M}_{n}(K)$ for some field extension $K$ of $F$, so $A / J \cong \mathrm{M}_{n}(K)$, where $J$ is the radical of $A$, and we know that $J^{\ell}=0$, and thus is "idempotent-lifting" by 23 , Corollary 1.1.28]. Then by [23, Proposition 1.1.25], $J=\mathrm{M}_{n}\left(J_{1}\right)$ for $J_{1} \triangleleft W$, and $J_{1}$ is nilpotent, implying $W_{1} / J_{1} \cong K$; thus $W$ is local.

Remark 2.33. Recall the sub-Peirce decomposition of [7, Definition 5.20] and [8, Remark 2.16], which is the restriction to $A$ of the Peirce decomposition of $K A$, namely

$$
A \subseteq \bigoplus A_{u v}^{\left(\mu \mu^{\prime}\right)}, \quad A_{u v}^{\left(\mu \mu^{\prime}\right)}=\bar{e}_{u}^{(\mu)} A \bar{e}_{v}^{\left(\mu^{\prime}\right)},
$$

where the sum ranges over $u, v=1, \ldots, k, \mu=1, \ldots, c_{u}$ and $\mu^{\prime}=1, \ldots, c_{v}$. This is not a decomposition of $A$, as the $A_{u v}^{\left(\mu \mu^{\prime}\right)}$ are contained in $K A$, but not in $A$ in general.

But the sub-Peirce decomposition reduces to the usual Peirce decomposition when the gluing of vertices is identical. Specifically, if $e_{r}=\sum e_{r, i}$ is the sum of identically glued vertices, then $e_{r} A e_{r} \cong \mathrm{M}_{n}\left(W_{r}\right)$, where $W$ is a local algebra over 
$F$. From now on, when we refer to Peirce components, we mean the ones along the diagonal, i.e., these algebras $e_{r} A e_{r}$.

Example 2.34. Let us illustrate the construction in Definition 2.29, where $C=F$. The full quiver $I \longrightarrow I I \longrightarrow I I I$ gives rise to the algebra

$$
\left\{\left(\begin{array}{ccc}
\alpha & \beta & \mu \\
0 & \gamma & \delta \\
0 & 0 & \nu
\end{array}\right): \alpha, \beta, \gamma, \delta, \mu, \nu \in F\right\} .
$$

On the other hand, the full quiver $I \longrightarrow I I \longrightarrow I I$ gives rise to the algebra

$$
\left\{\left(\begin{array}{ccc}
\alpha & \beta & \mu \\
0 & \gamma & \delta \\
0 & 0 & \gamma
\end{array}\right): \alpha, \beta, \gamma, \delta, \mu \in F\right\} .
$$

Here the diagonal Peirce components are $F$ and the local algebra

$$
\left\{\left(\begin{array}{ll}
\gamma & \delta \\
0 & \gamma
\end{array}\right): \gamma, \delta \in F\right\}
$$

The full quiver $I \longrightarrow I I \longrightarrow I I^{(2)}$ gives rise to the algebra

$$
\left\{\left(\begin{array}{ccc}
\alpha & \beta & \mu \\
0 & \gamma & \delta \\
0 & 0 & \gamma^{q^{2}}
\end{array}\right): \alpha, \beta, \gamma, \delta, \mu \in F\right\} .
$$

We can further refine the basic full quiver, by turning to relatively free algebras. Given the variety $V$ of a representable algebra, we can take the Zariski closure of its relatively free algebra $U$ and construct its full quiver $\Gamma$. (In fact, every variety is the variety of a representable algebra, but this is a deep theorem, due to Kemer [18] in characteristic 0 [17] over arbitrary infinite fields and to Belov [2] over finite fields and arbitrary commutative rings.) Then $\operatorname{id}(V)=\operatorname{id}(U)=\operatorname{id}(\mathcal{A}(\Gamma))$, so results about the relatively free algebra $U$ determine the identities of $V$.

Although this paper focuses on full quivers of relatively free PI-algebras, the connection can be rather tenuous.

Example 2.35. Consider the full quiver $\Gamma$ consisting of two paths of length 2, connecting the same two vertices. The algebra $\mathcal{A}(\Gamma)$ can be viewed as the subalgebra of upper triangular matrices generated by the matrix units $e_{1,2}, e_{1,3}, e_{2,4}, e_{3,4}$ and the $e_{i, i}, i=1,2,3,4 . \mathcal{A}(\Gamma)$ is not a relatively free PI-algebra since it does not contain a generic element in the (1,1)-position, but it does contain the relatively free algebra (without 1) generated by indeterminates $x_{1}, x_{2}, x_{3}, x_{4}$ satisfying $x_{1} x_{3}=x_{2} x_{4}$ and all other $x_{i} x_{j}=0$, since these are the only relations arising from the arrows.

2.7. The monoid grade on a full quiver. The algebra $\overline{F[\Lambda]}$ of Remark 2.30 can be viewed naturally as a $C$-algebra, and moreover is an $\left(\mathcal{M}_{r}, \mathcal{M}_{s}\right)$-bimodule, when $\mathcal{M}_{r}$ and $\mathcal{M}_{s}$ are suitably defined monoids. This gives a grading on $\overline{F[\Lambda]}$, according to the degrees of the monomials. We can refine this grading.

Definition 2.36. We write $\mathcal{M}_{\infty}$ for the multiplicative monoid $\left\{1, q, q^{2}, \ldots, \epsilon\right\}$, where $\epsilon a=\epsilon$ for every $a \in \mathcal{M}_{m}$. (In other words, $\epsilon$ is the "zero" element adjoined to the multiplicative monoid $\langle q\rangle.) \quad \mathcal{M}_{m}$ denotes the monoid obtained by adjoining a "zero" element $\epsilon$ to the subgroup $\langle q\rangle$ of $\mathbb{Z}_{q^{m}-1}$, namely $\mathcal{M}_{m}=$ $\left\{1, q, q^{2}, \ldots, q^{m-1}, \epsilon\right\}$, where $\epsilon a=\epsilon$ for every $a \in \mathcal{M}_{m}$. Let $\overline{\mathcal{M}}$ be the semigroup 
$\mathcal{M} / \sim$, where $\sim$ is the equivalence relation obtained by matching the degrees of glued variables: When two vertices have Frobenius gluing $\alpha \rightarrow \phi^{i}(\alpha)$, we identify 1 with $q^{i}$ in the respective components.

Lemma 2.37. Every $\mathcal{M}_{m}$ is a quotient of $\mathcal{M}_{\infty}$, and more generally whenever $m \mid m^{\prime}$, the natural group projection $\mathbb{Z}_{q^{m^{\prime}-1}} \rightarrow \mathbb{Z}_{q^{m}-1}$ extends to a monoid homomorphism $\mathcal{M}_{m^{\prime}} \rightarrow \mathcal{M}_{m}$.

Proof. One needs only to check that products involving the zero elements are preserved, but this is obvious since they are all zero.

Remark 2.38. Assume that $A=\mathcal{A}(\Gamma)$ is the algebra of a full quiver $\Gamma$.

(i) First suppose that $\Gamma$ is a branch, whose $r$ vertex is labelled $n_{r}\left(t_{r}\right)$. The diagonal blocks $S_{r}$ of $A$ (under multiplication) can be viewed as $\mathcal{M}_{t_{r}}$-modules, where we define the product $\left[q^{i}\right] a$ to be $a^{q^{i}}$, and $[\epsilon] a=0$.

When the base field $F$ is infinite, in (6) the full quiver can be separated (replacing $\alpha$ by $\alpha \gamma$ where $\gamma^{q} \neq \gamma$ ), and the algebra can be embedded in a graded algebra. In other words, each $S_{r}$ is $\mathcal{M}_{\infty}$-graded, where the indeterminates $\lambda_{\mathbf{i}}$ are given degree 1 ; hence, $A$ is naturally $\overline{\mathcal{M}}$-graded.

(ii) When the base field is finite (but the center of the block properly contains $F$ ), we must contend with Frobenius gluing. Homogeneous components of different degrees may be glued, so $S_{r}$ itself is not graded, and we need to pass to the larger algebra $B$ arising from the sub-Peirce decomposition of $A$; cf. Remark 2.33.

Let $A_{r, r^{\prime}}$ denote the $\left(r, r^{\prime}\right)$-sub-Peirce component of $A$. Then $A_{r, r^{\prime}}$ is naturally a left $F_{r}$-module and a right $F_{r^{\prime}}$-module, so $A_{r, r^{\prime}}$ is graded by the monoid $\mathcal{M}_{\hat{r}}$ obtained from $F_{\hat{r}}$, the compositum of $F_{r}$ and $F_{r^{\prime}}$. (In other words, if $F_{r}$ has $q^{t}$ elements and $F_{r^{\prime}}$ has $q^{t^{\prime}}$ elements, then $F_{\hat{r}}$ has $q^{\hat{t}}$ elements, where $\hat{t}=\operatorname{lcm}\left(t, t^{\prime}\right)$.) Since the free module is graded and the monoid $\mathcal{M}_{\hat{r}}$ is invariant under the Frobenius relations, we see that the Frobenius relations preserve the grade under $\mathcal{M}_{\hat{r}}$. Thus, the relatively free algebra of $\mathcal{A}(\Gamma)$ is graded with respect to $\mathcal{M}_{\hat{r}}$.

(iii) Given a full quiver $\Gamma$, let us write $\mathcal{U}(\Gamma)$ for the Zariski closure of the relatively free algebra of $\mathcal{A}(\Gamma)$. Suppose moreover that $\mathcal{B}$ is a branch of $\Gamma$, and $\mathcal{B}^{\prime}$ is a permuted branch of $\mathcal{B}$ with respect to some permutation. Applying (i) to $\mathcal{B}$ gives us an $\mathcal{M}(\mathcal{B})$-grading for the vertices of $\mathcal{U}(\mathcal{B})$, and likewise, we have an $\mathcal{M}\left(\mathcal{B}^{\prime}\right)$ grading for $\mathcal{U}\left(\mathcal{B}^{\prime}\right)$. However, the hypothesis that $\mathcal{B}^{\prime}$ is a permuted branch of $\mathcal{B}$ means there is a natural isomorphism $\Psi_{\mathcal{M}(\mathcal{B}), \mathcal{M}\left(\mathcal{B}^{\prime}\right)}: \mathcal{M}(\mathcal{B}) \rightarrow \mathcal{M}(\mathcal{B})$, by means of which we define homogeneous elements of $a \in A$ and $\mathcal{U}\left(\mathcal{B}^{\prime}\right)$ to be compatible if their grades correspond under this isomorphism. Clearly, glued diagonal elements of the respective blocks are compatible. Likewise, one can extend this isomorphism to (ii), and define compatible off-diagonal elements, and observe that glued offdiagonal elements must be compatible.

Example 2.39. Consider the example

$$
\left\{\left(\begin{array}{cc}
\alpha & \beta \\
0 & \alpha^{q}
\end{array}\right): \alpha \in \mathbb{F}_{q^{r}}, \beta \in K\right\}
$$

whose full quiver is $I \longrightarrow I^{(1)}$. The grading monoid in this case is $\mathcal{M}_{r} \times \mathcal{M}_{r}$ modulo the identification $(1, \epsilon) \equiv(\epsilon, q)$.

The sub-Peirce decomposition in this case has three components, one for each matrix entry, and the generic element $\left\{\left(\begin{array}{cc}x & y \\ 0 & x^{q}\end{array}\right)\right\}$ subdivides into the three 
components

$$
\left\{\left(\begin{array}{cc}
x & 0 \\
0 & 0
\end{array}\right)\right\}, \quad\left\{\left(\begin{array}{cc}
0 & 0 \\
0 & x^{q}
\end{array}\right)\right\}, \quad\left\{\left(\begin{array}{ll}
0 & y \\
0 & 0
\end{array}\right)\right\} .
$$

Note that $x$ and $x^{q}$ have the same grade.

Definition 2.40. The grade is called uniform if any two paths with the same initial and terminal vertex have the same grade.

Example 2.41. Suppose $F=\mathbb{F}_{q}$, so that $x^{q}-x \in \operatorname{id}(F)$. Consider the full quiver

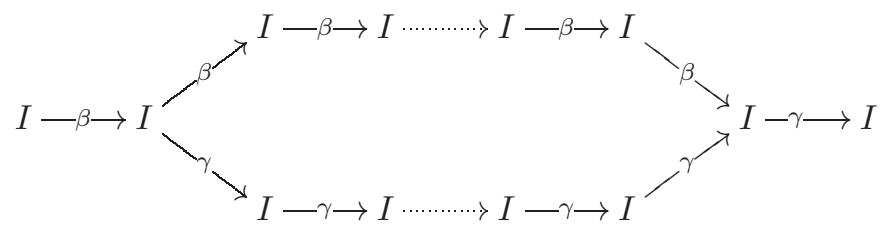

in which $\beta$ and $\gamma$ appear $q$ times each. In view of the Frobenius gluing of the arrows, the products of the arrows of the two branches have equal evaluations (coefficient $\beta^{q} \gamma=\beta \gamma=\beta \gamma^{q}$ ) in representations over $F$, even though no two subpaths between the same two vertices have the same grade.

\section{Canonization theorems}

The object of this section is to reduce full quivers to a better form.

Definition 3.1. A subdirect decomposition $\Gamma=\bigcup \Gamma_{i}$ of full quivers is a union of subquivers $\Gamma_{i}$ such that, for any arrow of $\Gamma_{i}$, all arrows of $\Gamma$ glued to this arrow also appear in $\Gamma_{i}$.

A pseudo-quiver is subdirectly decomposable if it admits a proper subdirect decomposition, and subdirectly indecomposable otherwise.

Definition 3.2. A full quiver (resp. pseudo-quiver) is basic if it has a unique initial vertex $r$ and unique terminal vertex $s$. A basic full quiver (resp. pseudo-quiver) $\Gamma$ is canonical if it has vertices $r^{\prime}$ and $s^{\prime}$ satisfying the following properties:

- $\Gamma$ starts with a unique path $p_{0}$ from $r$ to $r^{\prime}$. Thus, every vertex not on $p_{0}$ succeeds $r^{\prime}$.

- $\Gamma$ ends with a unique path $p_{0}^{\prime}$ from $s^{\prime}$ to $s$. Thus, every vertex not on $p_{0}^{\prime}$ precedes $s^{\prime}$.

- Any two paths from the vertex $r^{\prime}$ to the vertex $s^{\prime}$ have the same grade.

An enhanced canonical full quiver (resp. pseudo-quiver) is a canonical full quiver (resp. pseudo-quiver) with uniform grade.

Proposition 3.3. When the full quiver or pseudo-quiver of an algebra $A$ is canonical, a representation can be chosen such that there is no identical gluing among edges originating in $r^{\prime}$ or terminating in $s^{\prime}$.

Proof. Each gluing between two edges initiating at the vertex $r^{\prime}$ must correspond to a quasi-linear relation. Multiplying by any element of $A$ must preserve this relation, implying diagonal gluing between edges originating at $r^{\prime}$; thus, there is degenerate gluing which we eliminate via Proposition 3.16. Thus, we have removed all gluing on edges originating at $r^{\prime}$. Note that this procedure does not affect subsequent edges, so we can perform the same procedure on the dual quiver and remove all gluing on edges terminating at $s^{\prime}$. 
Theorem 3.4. Any representable algebra $A$ is a subdirect product of algebras having basic full quivers.

Proof. Suppose the algebra $A$ has full quiver $\Gamma$. For every branch $\mathcal{B}$ of $\Gamma$, say with initial vertex $r$ and terminal vertex $s$, let $\Gamma_{r, s}$ denote the subquiver comprised of all subpaths of paths whose initial vertex is $r$ and terminal vertex is $s$. We claim that $\Gamma_{r, s}$ is convex. Indeed, for any two paths $p_{1}, p_{2}$ whose concatenation $p_{1} p_{2}$ is a subpath of $\Gamma_{r, s}$, we can extend $p_{1}$ to a path starting in $r$ and $p_{2}$ to a path ending in $s$, and thus each is a subpath of a path from $r$ to $s$.

Thus, by Proposition 2.9, there is a projection $A \rightarrow A_{\Gamma_{r, s}}$. But any arrow of $\Gamma$ is in some $\Gamma_{r, s}$. Hence an element of $A$ is in the kernel iff it annihilates all the arrows of $\Gamma$, which means that the natural map $A \rightarrow \prod_{r, s} A_{\Gamma_{r, s}}$ is an injection.

3.1. The Second Canonization Theorem. We recall the following notion from [8].

Definition 3.5. A polynomial (resp. power series) of the form $\sum_{i} \sum_{j>1} c_{i j} \lambda_{i}^{q_{i j}}$ is called a $q$-polynomial if each $q_{i j}$ is a $q$-power with $q=|F|$, where we take $q=1$ if $F$ is infinite.

We need [8, Proposition 6.5]:

Proposition 3.6. Suppose $V_{0}$ is an irreducible quasi-linear affine variety (containing 0). Then there exist independent variables $\lambda_{i_{1}}, \ldots, \lambda_{i_{s}}$, such that all other $\lambda_{i}$ can be expressed in terms of q-power series in $\lambda_{i_{1}}, \ldots, \lambda_{i_{s}}$.

Theorem 3.4 can be combined with the next result:

Theorem 3.7 (Second Canonization Theorem). Any basic full quiver $\Gamma$ (resp. pseudo-quiver) of a representable relatively free algebra can be modified (via a change of base) to an enhanced canonical full quiver (resp. pseudo-quiver) of an isomorphic algebra (i.e., relatively free algebra of the same variety).

Proof. We take $A$ to be the algebra of generic elements constructed from $\Gamma$. We need to decompose $A$ into a subdirect product of algebras having enhanced canonical full quivers.

First assume that $F$ is infinite. In this case the only gluing is proportional, and any gluing between arrows initiating at $e_{r}$ must correspond to a relation of the form $\sum \alpha_{i} a_{r, s_{i}}=0$. Multiplying by any element of $A$ must preserve this relation, implying diagonal gluing between the various $s_{i}$ blocks; thus, there is degenerate gluing between the $B_{r, s_{i}}$, which we can eliminate by means of Proposition 3.16.

For $F$ finite, we must also take Frobenius gluing into account, and we need the more elaborate straightening argument of [8, Theorems 6.12], which we review here. Let $p=\operatorname{char}(F)$. By [7, Theorem 7.10], the Zariski closure of $A$ has finite PI-rank, which means that it is PI-equivalent to a representable affine PI-algebra. Consider the algebra $\tilde{A}$ generated by its generic elements and pass to its Zariski closure $\hat{A}$. In view of [8, Lemma 6.1], we can decompose $J=V_{0} \oplus V_{1}$ as a direct sum of the connected component $V_{0}$ of 0 and a space $V_{1}$ which is finite dimensional over $F$ and thus finite, say with base $b_{1}, \ldots, b_{m}$. We take $\tilde{A}$ generated by $V_{0}$ and all elements $w_{k}=\sum_{j} \psi_{j, k} b_{j}$ of $V_{1}, 1 \leq k \leq m$, where $\psi_{j, k} \in F$. Note that $\psi_{j, k}^{q}=\psi_{j, k}$ since $|F|=q$. Then any element of $\tilde{A}$ can be represented as a linear combination of products of length $\leq \ell m$ of these elements. 
We project onto the variety $\bar{V}$ defined by $q$-polynomials of degree $\leq q^{\ell m}$. In 7 , Theorem 4.10], it is proved that the ideal of polynomial relations of an $F$-vector space $A$ is generated by $q$-polynomial relations. Thus, some subset of the arrows is independent, and all other arrows can be written as $q$-polynomials in terms of these arrows. We are now in a position to repeat the previous argument, but this time our relations are in terms of $q$-polynomials rather than linear combinations. Thus, in this case, we only reduce to proportional Frobenius gluing.

Corollary 3.8. Any relatively free representable algebra $A$ is a subdirect product of algebras with representations whose full quivers $\Gamma_{i}$ are canonical. In particular, $\operatorname{id}(A)=\bigcap_{i} \operatorname{id}\left(\Gamma_{i}\right)$.

Proof. The subdirect decomposition of Theorem 3.4 can be done at the level of varieties, i.e., of relatively free algebras, so Theorem 3.7 is applicable. The last assertion follows at once.

Thus, the quiver theory of PI-algebras can be reduced to the case of enhanced canonical full quivers. Accordingly, all of the full quivers and pseudo-quivers that we consider in this paper will be enhanced canonical. Quivers tell us how our algebra is represented, whereas, as we shall see, pseudo-quivers tell us about evaluations of monomials. These two notions combine when we consider relatively free PI-algebras below.

3.2. The Third Canonization Theorem. In this subsection we prove the Third Canonization Theorem. The purpose of this theorem is to show how quiver reductions affect the T-ideals of the corresponding relatively free algebras. Accordingly, we only consider a certain kind of T-ideals, but get very explicit information for them.

Definition 3.9. A T-ideal $\mathcal{I}$ of a representable algebra $A$ with a given representation $\rho: A \mapsto \mathrm{M}_{n}(C)$ (for $C$ Noetherian, perhaps an extension of $K$ ) is $C$-closed if it is closed under multiplication by elements of $C$.

The next lemma, which serves as a converse to Remark 2.31, plays an important role both in this paper and in 10 .

Lemma 3.10. Suppose $A$ is a relatively free PI-algebra with pseudo-quiver $\Gamma$ with respect to a representation $\rho: A \mapsto \mathrm{M}_{n}(C)$, and $\mathcal{I}=\mathrm{id}(A)$ is a $C$-closed $T$-ideal. Then $A$ is PI-equivalent to the algebra $A^{\prime}$ of the pseudo-quiver $\Gamma$.

Proof. Clearly id $(A) \supseteq \operatorname{id}\left(A^{\prime}\right)$, since in constructing $A^{\prime}$ we are recovering elements corresponding to the elements of $A$. Let $\bar{A}$ denote the Zariski closure of $A$, and let $\tilde{A}$ denote the subalgebra of $\bar{A}$ generated by generic elements, cf. 8, Construction 7.14]. Then $\operatorname{id}(\tilde{A}) \supseteq \operatorname{id}(\bar{A})=\operatorname{id}(A)$. The conditions of the lemma show that $\tilde{A}$ is relatively free, by [8, Theorem 7.15]. But any set of elements of $A^{\prime}$ then has a pre-image in $\tilde{A}$, so $\operatorname{id}\left(A^{\prime}\right) \supseteq \operatorname{id}(\tilde{A})$. Putting everything together yields

$$
\operatorname{id}(\tilde{A}) \supseteq \operatorname{id}(\bar{A})=\operatorname{id}(A) \supseteq \operatorname{id}\left(A^{\prime}\right) \supseteq \operatorname{id}(\tilde{A}),
$$

yielding equality at each stage, as desired.

Corollary 3.11. Suppose $A$ is a relatively free PI-algebra. If $C^{\prime}$ is a faithful $C$ algebra, then the algebra of the pseudo-quiver of the natural representation into $\mathrm{M}_{n}\left(C^{\prime}\right)$ is also PI-equivalent to $A$. 
Proof. Passing to $C^{\prime}$ does not change the identities.

Theorem 3.12. Suppose $A$ is a relatively free PI-algebra having a representation with pseudo-quiver $\Gamma$, and $\mathcal{I} \neq 0$ is a T-ideal of $A$ closed under multiplication by characteristic coefficients of the elements of $A$ under the given representation. Then $A^{\prime}=A / \mathcal{I}$ is obtained by means of the following elementary operations:

(1) New relations on the base ring and its pseudo-quiver $\Gamma^{\prime}$ are obtained by the appropriate new gluing. This means:

- Gluing, perhaps up to infinitesimals (which means introducing relations as in equation (10), with or without a Frobenius twist. (This involves relations of the form $\lambda_{i}-\lambda_{j}$ or $\lambda_{i}^{q}-\lambda_{j}$. Note that when $i=j$, the gluing is of a block with itself; when this occurs with a Frobenius twist, the block must become finite.)

- New quasi-linear relations on arrows, perhaps up to infinitesimals.

- Reducing the matrix degree of a block attached to a vertex. (This is achieved by adjoining every characteristic coefficient for the HamiltonCayley relations of smaller degree, and, for Frobenius relations, relations of the form

$$
c^{q} f\left(a_{1}, \ldots, a_{m}\right)-c f\left(a_{1}, \ldots, a_{m}\right),
$$

for every characteristic coefficient.)

(2) New linear dependences on vertices (which could include cancelling extraneous vertices) between which any two paths must have the same grade.

In particular, $A^{\prime}$ satisfies a pseudo-quiver relation not satisfied by $A$. (Although this theorem is formulated for pseudo-quivers, we recall that over an infinite field the pseudo-quiver theory reduces to the theory of full quivers, in view of Theorem 2.12.) Note that the new gluing could lower the nilpotence index of the Jacobson radical. New relations on the base ring will be seen in the pseudo-quiver via new relations on arrows or on the vertices.

In a subsequent paper we will prove that any nonzero T-ideal contains a $C$-closed T-ideal for some affine commutative $F$-algebra $C$ over which $A$ is representable. Theorem 3.12 says that in passing from one variety to another, we obtain the finite direct product of relatively free algebras whose pseudo-quivers are obtained from the initial pseudo-quiver by the elementary processes described in the statement of the theorem. Gluing up to infinitesimals could increase the nilpotence index of the radical, and gluing of vertices could impose new relations between branches, such as connecting branches which had been previously disjoint.

Although this theorem is not needed in the proof of Specht's conjecture for affine PI-algebras over arbitrary Noetherian rings, it does show the relevance of full quivers to PI-theory. Its proof is based on the following notion.

Definition 3.13. For a module $M$ over a commutative Noetherian ring $C$, an element $c \in C$ is $M$-torsion if for each $a \in M$ there is $k=k(a)>0$ such that $c^{k} a=0$. If there is some $k$ such that $k=k(a)$ for all $a \in M$, we call this the torsion index for $c$. We define $\operatorname{tor}_{C}(M)$ to be $\{c \in C: c$ is $M$-torsion $\}$.

It is well known that $\operatorname{tor}_{C}(M)$ is an ideal of $C$.

Lemma 3.14. Suppose $C$ is a commutative ring. For any Noetherian $C$-module $M, \operatorname{tor}_{C}(M)$ has bounded torsion index. 
Proof. Define $\operatorname{tor}_{C}(M)_{k}=\left\{c \in \operatorname{tor}_{C}(M): c\right.$ has torsion index $\left.k\right\}$. Then the series $\operatorname{tor}_{C}(M)_{1} \subseteq \operatorname{tor}_{C}(M)_{2} \subseteq \cdots$ stabilizes.

We now prove Theorem 3.12 .

Proof. In view of [7] Theorem 7.20 and Corollary 7.21], the variety of any representable algebra $A$ has a relatively free algebra that is affine. Thus, we may assume that $A$ is affine, and its generators are generic elements. We take $\hat{C}$ as in Remark 1.1. Since in characteristic $p$ one sometimes wants to employ the trick that every matrix has a $\bar{q}$ power that is semisimple for some $\bar{q}$, we take $\hat{C}_{0}=\hat{C}^{\bar{q}}$, a radical extension of $\hat{C}_{0}$; cf. [6, Remark 2.35 and Lemma 2.36], for example, which is used in Lemma 5.1. We localize $\hat{A}=\left(A \otimes_{C} \hat{C}_{0}\right) / \mathcal{I}$ with respect to characteristic coefficients arising in Shirshov's Theorem.

There is a natural injection $\hat{A} \rightarrow \oplus \hat{A}_{\langle c\rangle} \oplus\left(\oplus \hat{A} /\left\langle c^{j}: c \in \hat{C}_{0}, j<k\right\rangle\right)$. By Lemma 3.14 since $\mathcal{I} \neq 0$, each component on the right side must have torsion, and thus have a new pseudo-quiver which thereby produces a new relation from the list. But this produces a pseudo-quiver with either new gluing, central polynomial of lower degree, or new identification of arrows, and this process must terminate after a finite number of steps.

3.3. Removing degenerate gluing. In the case of degenerate gluing (see Definition [2.6), the two branches produce the same values when we multiply out the elements in the corresponding algebra. When we take the (weighted) difference in the products, then the two branches may cancel, as illustrated in the following full quiver:

\section{Example 3.15.}

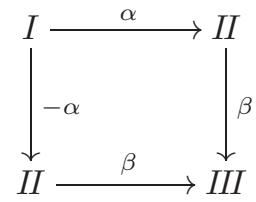

Since all evaluations along the whole branches cancel out, we are left only with evaluations for partial branches. Consequently, this full quiver is PI-equivalent to the full quiver with two disconnected branches:

$$
I \longrightarrow I I ; \quad I I \longrightarrow I I I \text {. }
$$

In [8, Remark 5.18 and Proposition 5.20] we saw how to eliminate degenerate gluing, but at the cost of passing to an arbitrary commutative affine base ring. Nevertheless, the same argument enables us to reduce the algebra of the full quiver to a semi-direct product of algebras of subquivers.

Proposition 3.16. Any representable, relatively free algebra is PI-equivalent to an algebra whose full quiver has no degenerate gluing.

Proof. Take the Zariski closure $\hat{A}$ of $A$. By the Second Canonization Theorem, Theorem 3.7 we may assume that the quiver has a unique initial vertex $v_{1}$. We repeat the argument of [8, Theorems 6.10 and 6.12] (slightly abbreviated), which might be difficult to extract from there:

Claim: We may assume that some power of each indeterminate is 0 . 
To prove the claim, first assume that the base field $F$ is infinite. Then $\hat{A} \otimes_{F} C$ is PI-equivalent to $\hat{A}$, so we may work in $\hat{A} \otimes_{F} C$. We pass to the representation of $\hat{A}$ as transformations of $V^{\prime}=V \otimes_{F} C$, where the $\widehat{\theta_{i, u}}$ are commuting indeterminates over $F$ and $C=F\left[\widehat{\theta_{i, u}}\right] /\left\langle\widehat{\theta_{i, u}}\right\rangle^{\ell}$. (Since we have only finitely many $\theta_{i, u}$ needed for our finitely many relations, we see that $[C: F]<\infty$, and thus $\left[V^{\prime}: K\right]<\infty$.) Let $\bar{\theta}_{i, u}$ denote the canonical image of $\widehat{\theta_{i, u}}$ in $C$. The nonzero products $\tilde{\theta}$ of the $\bar{\theta}_{i, u}$ comprise a base $\Theta$ for $C$ over $F$; if $B$ is a base for $V$, then $B \otimes \Theta$ is a base for $V^{\prime}$ over $F$, of order $n^{\prime}=n[C: F]$.

Thus, we may assume that the base field $F$ is finite, of characteristic $p$. By [7. Theorem 7.10], $\hat{A}$ has finite PI-rank, which means that it is PI-equivalent to a representable affine PI-algebra. Replace $A$ by the algebra $\tilde{A}$ generated by its generic elements ([7, Theorem 7.15]), and pass to its Zariski closure $\hat{A}$.

Let $V_{0}$ be the connected component of 0 in $J . J / V_{0}$ is a finite set, and also a vector space over $F$, and thus has some finite base $\bar{b}_{1}, \ldots, \bar{b}_{k}$, which we lift back to $b_{1}, \ldots, b_{k} \in V . V_{1}=\sum F b_{i}$ is annihilated by $p=\operatorname{char} F$, and is a subvariety since $J$ is quasi-linear, so we have split $V J$ as $V_{0} \oplus V_{1}$.

We take $\tilde{A}$ generated by $V_{0}$ and all elements $w_{k}=\sum_{j} \psi_{j, k} b_{j}$ of $V_{1}, 1 \leq k \leq$ $m$, where $\psi_{j, k} \in F$. Note that $\psi_{j, k}^{q}=\psi_{j, k}$ since $|F|=q$. Then any element of $\tilde{A}$ can be represented as a linear combination of products of length $\leq \ell m$ of these elements. We need to replace the $w_{k}$ by generic elements $\sum_{j} \overline{\psi_{j, k}} b_{j}$ of $V_{1}$, where $\overline{\psi_{j, k}}$ are generic coefficients satisfying $\overline{\psi_{j, k}} q=\overline{\psi_{j, k}}$. These elements belong to the finite dimensional extension $F\left[\widehat{\psi_{j, k}}\right] /\left\langle\widehat{\psi_{j, k}}-\widehat{\psi_{j, k}}\right\rangle$, where $\widehat{\psi_{j, k}}$ are commuting indeterminates over $F$.

We project onto the variety $\bar{V}$ defined by $q$-polynomials of degree $\leq q^{\ell m}$. Since the projection of a quasi-linear variety is quasi-linear, we may work in $\bar{V}$, in which all our $q$-power series project to $q$-polynomials. In view of Proposition 3.6, there is some subset of the arrows which is independent, and all other arrows can be written as $q$-polynomials in terms of these arrows.

Again we pass to the representation of $\hat{A}$ over

$$
\left(J \otimes_{F} F\left[\theta_{i, j}\right] /\left\langle\theta_{i, j}\right\rangle^{\ell}\right) \oplus\left(F\left[\widehat{\psi_{j, k}}\right] /\left\langle{\widehat{\psi_{j, k}}}^{q}-\widehat{\psi_{j, k}}\right\rangle\right)
$$

we can eliminate arrows as in the case for $F$ infinite (by the same technique of expanding the graph and labelling one arrow of each relation as "dispensable"), but this time our relations are in terms of $q$-polynomials rather than linear combinations. Thus, in this case, we can only reduce to proportional Frobenius gluing. This proves the claim.

If the products of the primitive arrows sum to a nonzero map, replace the glued paths by a single branch having the same attributes. Otherwise, if the sum is zero, attach a formal commuting indeterminate $\widehat{\theta_{i, u}}$ to each vertex of an arrow.

Thus, each new vertex has the form of the tensor product of an old vertex and a monomial in the $\widehat{\theta_{i, u}}$. Since the algebra is generic, we may erase any duplication of arrows. We conclude via induction on the length of $\Gamma$.

Thus, we assume from now on that our full quivers do not have degenerate gluing. 


\section{IDENTITIES AND NONIDENTITIES OF FULL QUIVERS}

We have now arrived at one of the core issues: How do we translate the graphtheoretic information given in a full quiver into an identity or nonidentity that will encode enough information to determine the original full quiver? In order to characterize different kinds of full quivers of representations according to their identities, we first need to establish, for every full quiver in $\mathcal{C}$, either a corresponding polynomial identity or a nonidentity.

Our goal then is to find natural maps

$$
\Phi, \Psi \text { : Canonical full quivers } \rightarrow F\{X\},
$$

which provide "meaningful" information about id $(\Gamma)$. By "natural" we mean that $\Phi$ and $\Psi$ should be readily calculable from $\Gamma$. By "meaningful" we would like $\Phi(\Gamma) \notin$ $\operatorname{id}(\Gamma)$, whereas $\Psi(\Gamma) \in \operatorname{id}(\Gamma)$.

This section is composed mainly of examples, to provide some intuition. One hits a snag with Example 4.16 below, in which $\operatorname{id}\left(\Gamma_{1}\right)=\operatorname{id}\left(\Gamma_{2}\right)$ although $\Gamma_{1}$ and $\Gamma_{2}$ do not share the same geometrical makeup. So formally, we should modify our search and request that $\Psi\left(\Gamma_{1}\right)=\Psi\left(\Gamma_{2}\right)$ only when the full quivers $\Gamma_{1}$ and $\Gamma_{2}$ correspond to algebras that are PI-equivalent. Also, we will want $\Phi$ to reflect the gluing in $\Gamma$. Edges of the full quiver are sent to indeterminates, with linear gluing among the edges sent to linear relations among the indeterminates.

It turns out that $\Phi$ is more useful in the theory. We distinguish these according to the properties of $\Gamma$. Let us pinpoint the transition from $\Gamma$ to polynomial evaluations.

Remark 4.1. Given a monomial $f=x_{1} x_{2} \ldots x_{m}$, one can evaluate $f$ in an algebra $A$ along a path of its full quiver $\Gamma$ by specializing $x_{1}$ to an element $a_{1}$ corresponding to the first vertex, specializing $x_{2}$ to an element $a_{2}$ corresponding to the edge emanating from the first vertex, specializing $x_{3}$ to an element $a_{3}$ corresponding to the next vertex, and so forth. By the usual "staircase" argument, one would expect any evaluation along a different path to permute these substitutions and thus yield 0 . However, gluing complicates this computation, and in particular, degenerate gluing could produce other nonzero evaluations which cancel out the original evaluation, and yield $f\left(a_{1}, a_{2}, a_{3}, \ldots\right)=0$. We could circumvent this problem by means of Proposition [3.16, but at the cost of replacing the base field $F$ by a commutative affine algebra.

On the other hand, this phenomenon will not occur for pseudo-quivers, since degenerate gluing does not exist for pseudo-quivers (since radical elements can then be viewed as operators). The price is that we now have new relations on the vertices, as indicated in Remark 2.7.

4.1. Nonidentities of paths. For the remainder of this paper, our algebra $C$ of Definition 2.29 (over which we build the algebra from the full quiver) is the base field $F$. In this section we construct our nonidentity in the important case where the full quiver is a path; i.e., it has only one branch. In this case, $\Phi$ will send paths $\Gamma$ to nonidentities of $\mathcal{A}(\Gamma)$.

Definition 4.2. Let $\mathcal{C}$ be a class of enhanced canonical full quivers, and suppose $\Gamma \in \mathcal{C}$ is a full quiver. A polynomial $f$ is called a $\mathcal{C}$-critical nonidentity for $\Gamma$, if $f \notin \operatorname{id}(\Gamma)$, but $f \in \operatorname{id}\left(\Gamma^{\prime}\right)$ for every proper subquiver $\Gamma^{\prime}<\Gamma$.

Later we shall also require that the polynomial $\Phi(\Gamma)$ is "trace-absorbing" with regards to $\mathcal{A}(\Gamma)$, as defined in Definition 5.3. Our selection is not based on degree, 
but rather on combinatorial considerations which will become clear. We focus on nonidentities which force specific sorts of substitutions to obtain nonzero evaluations of $f$.

Our approach is as follows:

- We construct $\Phi$ on paths $\Gamma=\mathcal{B}$. In this case. $\Phi(\Gamma)$ is a critical nonidentity. We could pass to the canonical pseudo-quiver, since it is easier to compute with this, but we stay with full quivers since their formal properties are more straightforward. The main complication in this step is nonidentical Frobenius gluing, which requires special treatment.

- Later, we treat permuted canonical full quivers $\Gamma$. We define $\Psi(\Gamma)$ to be a blended PI of $\mathcal{A}(\Gamma)$, obtained as a weighted sum of $\Phi$ applied to the branches, with coefficients obtained from the gluing.

- We perform the reduction of arbitrary full quivers to permuted canonical full quivers, which is parallel to writing a polynomial as a sum of blended polynomials.

We always take the polynomial $\Phi(\Gamma)$ to be quasi-linear with respect to $\mathcal{A}(\Gamma)$. By Remark 2.22. in order to check whether $f \in \operatorname{id}(\Gamma)$, one need only consider semisimple and radical substitutions.

Lemma 4.3. A nonzero evaluation of a blended polynomial lies in a diagonal Wedderburn block iff each substitution of the indeterminates is semisimple.

Proof. $(\Rightarrow)$ Multiplying by a radical substitution moves us along to a subsequent block.

$(\Leftarrow)$ Multiplying by a semisimple substitution leaves us in the same block (i.e., the same vertex).

Lemma 4.4. In the absence of gluing between branches, identities are determined by evaluations on branches.

Proof. Since full quivers of representations lack cycles, any nonzero evaluation of a monomial must occur by specializing elements to a path.

On the other hand, gluing between branches leads to cancellation of terms and provides the PIs which are the ones of greatest interest to us.

An important role is played by Lewin's Theorem [20]:

$$
\operatorname{id}\left(\begin{array}{cc}
R_{1} & * \\
0 & R_{2}
\end{array}\right)=\operatorname{id}\left(R_{1}\right) \operatorname{id}\left(R_{2}\right) \text {. }
$$

Recall that the length of a branch $\mathcal{B}$ is its number of arrows, which equals its number of vertices, minus 1 . A typical path of length $\ell$ can be written in the form

$$
\bullet_{n_{1}\left(t_{1}\right)} \rightarrow \bullet_{n_{2}\left(t_{2}\right)} \rightarrow \cdots \rightarrow \bullet_{n_{\ell+1}\left(t_{\ell+1}\right)} \text {. }
$$

Proposition 4.5. Any nonzero product of $k$ radical elements of $A$ must correspond to $k$ edges in a path, and thus the full quiver of such an algebra A must have a branch of length $\geq k$. More generally, if

$$
f_{1}\left(x_{1,1}, \cdots, x_{1, n_{1}}\right) y_{1} f_{2}\left(x_{2,1}, \cdots, x_{2, n_{2}}\right) y_{2} \cdots y_{\ell} f_{\ell+1}\left(x_{\ell+1,1}, \cdots, x_{\ell+1, n_{\ell+1}}\right)
$$

is a blended polynomial evaluated on an algebra whose radical is of nilpotence index $\ell+1$, where the $f_{j}$ are polynomials in indeterminates $x_{j, 1}, x_{j, 2}, \ldots$, and if each $y_{i}$ is specialized to a radical substitution, then each nonzero evaluation must come from semisimple substitutions for all of the $x_{j, 1}, x_{j, 2}, \ldots$. 
Proof. The first assertion is immediate. But then all the other substitutions must be semisimple, and thus the second assertion follows from Lemma 4.3 .

4.1.1. Digression: Identities of matrix algebras over finite fields. For motivation, we start with the easiest full quivers, namely when the full quiver is comprised just of a single vertex. Since this corresponds to a matrix algebra over a field, we pause for a thorough review of the identities of finite $n \times n$ matrix algebras.

Here is a subtlety even for $n=1$.

Example 4.6. Consider the finite fields $\mathbb{F}_{q^{t}}$ and their algebraic closure $\overline{\mathbb{F}}_{q}$. Note that $\left\{\operatorname{id}\left(\mathbb{F}_{q^{t}}\right)\right\}$ is a decreasing chain of T-ideals, and that $\operatorname{id}\left(\overline{\mathbb{F}}_{q}\right)$ is its intersection. It follows that $\overline{\mathbb{F}}_{q}$ cannot be distinguished from all the $\mathbb{F}_{q^{t}}$ via a finite set of identities (this follows indeed from the compactness theorem). Of course, we can distinguish among the various $\left\{\operatorname{id}\left(\mathbb{F}_{q^{t}}\right)\right\}$ by means of the polynomials $x^{q^{t}}-x$.

Proposition 4.7. If $|F|=q$, then $\mathrm{M}_{n}(F)$ satisfies the one-variable identity $x^{p^{u} q^{m}-}$ $x^{p^{u}}$, where $p=\operatorname{char}(F), p^{u} \geq n$, and $m$ is the least common multiple of the numbers $\leq n$.

Proof. Taking $E$ to be the field of order $q^{m}$, any matrix in $\mathrm{M}_{n}(F)$ can be viewed in $\mathrm{M}_{n}(E)$ as the sum of a semisimple matrix and a nilpotent matrix which commute with each other, and taking the $p^{u}$ power kills the nilpotent part; thus the assertion is seen by checking the components of an arbitrary diagonal matrix.

Our next question (whose answer is immediate over infinite fields): How do we distinguish between $\operatorname{id}\left(\mathrm{M}_{n_{1}}\left(\mathbb{F}_{q_{1}}\right)\right)$ and $\operatorname{id}\left(\mathrm{M}_{n_{2}}\left(\mathbb{F}_{q_{2}}\right)\right)$ ? By the theorem of Oates and Powell [21, every variety of finite rings is finitely based, but here we look for a special set of identities and nonidentities to differentiate between these algebras.

Remark 4.8. (i) For $n_{1}<n_{2}$, the polynomial

$$
h_{n, t}=\prod_{j=1}^{t-1} h_{n}^{q^{j}}-h_{n},
$$

where $h_{n}$ is as in Definition 2.15] is in $\operatorname{id}\left(\mathrm{M}_{n}\left(\mathbb{F}_{q^{m}}\right)\right)$ iff $m<t$.

(ii) For $n_{1}<n_{2}$, clearly $h_{n_{2}} \in \operatorname{id}\left(\mathrm{M}_{n_{1}}\left(\mathbb{F}_{q_{1}}\right)\right) \backslash \operatorname{id}\left(\mathrm{M}_{n_{2}}\left(\mathbb{F}_{q_{2}}\right)\right)$, but it is more difficult to determine if there exists $f \in \operatorname{id}\left(\mathrm{M}_{n_{2}}\left(\mathbb{F}_{q_{2}}\right)\right) \backslash \operatorname{id}\left(\mathrm{M}_{n_{1}}\left(\mathbb{F}_{q_{1}}\right)\right)$. For example, it could be that $\mathrm{M}_{n_{1}}\left(\mathbb{F}_{q_{1}}\right) \subseteq \mathrm{M}_{n_{2}}\left(\mathbb{F}_{q_{2}}\right)$. (This happens when $q_{1}$ divides $q_{2}^{n_{2} / n_{1}}$, seen as a consequence of the regular representation.)

In the notation of Remark 2.16, $f(A)^{+}$is closed under multiplication by coefficients of the characteristic polynomial of any simple component of an element (represented as a matrix). In particular, we could take $f$ to be either the Capelli polynomial, or if we want to rely on the easier theorem [6. Theorem J, p. 27], we could take instead the central polynomial $h_{n}$.

4.2. Elementary full quivers. The next most basic kind of full quiver, called elementary, is a full quiver that consists of a single edge. The underlying general ideas of the theory can already be seen in the case of an elementary full quiver, so we investigate the PIs of their algebras.

Recall that the full quiver $I_{n_{1}\left(t_{1}\right)} \rightarrow I I_{n_{2}\left(t_{2}\right)}$ corresponds to the algebra

$$
A=\left(\begin{array}{cc}
\mathrm{M}_{n_{1}}\left(F_{1}\right) & L \\
0 & \mathrm{M}_{n_{2}}\left(F_{2}\right)
\end{array}\right),
$$


where $F_{1}, F_{2}$ are intermediate subfields of $F \subseteq K$ which are either finite or equal to $K$, and $L$ is an $\left(\mathrm{M}_{n_{1}}\left(F_{1}\right), \mathrm{M}_{n_{2}}\left(F_{2}\right)\right)$-bisubmodule of the upper right part of $\mathrm{M}_{n_{1}+n_{2}}(K)$. Lewin's Theorem [20] implies that $\mathrm{id}(A)=\mathrm{id}\left(\mathrm{M}_{n_{1}}\left(F_{1}\right)\right) \operatorname{id}\left(\mathrm{M}_{n_{2}}\left(F_{2}\right)\right)$. Since we also treat algebras without 1 , it is possible that one or both of the vertices is empty, i.e., that the corresponding diagonal block is empty. Incidentally, if $F_{1}$ is finite whereas $F_{2}$ is infinite, then $\operatorname{id}(A)$ is not equal to the T-ideal of identities of any finite dimensional $F$-algebra; cf. 3. This phenomenon is in contrast to a theorem of Kemer for algebras over an infinite base field.

Example 4.9. There are three possibilities for an elementary full quiver:

(1) $\circ \rightarrow \circ$ yields nonzero entries only in the upper right corner of $\left(n_{1}+n_{2}\right) \times$ $\left(n_{1}+n_{2}\right)$ matrices. The corresponding T-ideal of identities is generated by the identity $x y$.

$(2) \circ \rightarrow \bullet$ corresponds to the algebra $\left\{\left(\begin{array}{ll}0 & * \\ 0 & *\end{array}\right)\right\}$. (We adopt the convention throughout that the entries denoted $*$ are arbitrary and not necessarily equal.) Its T-ideal of identities is $\left\{x f: f \in \operatorname{id}\left(\mathrm{M}_{n}(F)\right)\right\}$.

This easy special case of Lewin's theorem can be seen directly as follows: Consider a multilinear polynomial $g\left(x_{1}, \ldots, x_{d}\right)=\sum x_{i} f_{i}$, where $x_{i}$ does not appear in $f_{i}$. If $f_{i} \notin \operatorname{id}\left(\mathrm{M}_{n}(F)\right)$, then $x_{i} \mapsto e_{12}$ gives a nonzero specialization of $g$; if each $f_{i} \in \operatorname{id}\left(\mathrm{M}_{n}(F)\right)$, then $g$ is an identity.

Similarly, $I_{n(t)} \rightarrow \circ$ corresponds to $\left\{\left(\begin{array}{ll}* & * \\ 0 & 0\end{array}\right)\right\}$, and the corresponding variety is $\left\{f x: f \in \operatorname{id}\left(\mathrm{M}_{n}(F)\right)\right\}$, and has the critical nonidentity $c_{n^{2}}\left[x, h_{n}\right]$.

(3) When both vertices are nonempty, the full quiver $I_{n_{1}\left(t_{1}\right)} \rightarrow I I_{n_{2}\left(t_{2}\right)}$ has the critical nonidentity $c_{n_{1}^{2}}\left[x, h_{n}\right] c_{n_{2}^{2}}$, where $n=\max \left\{n_{1}, n_{2}\right\}$.

Next, recall the definition of compression from Remark 2.4. When a branch compresses to a point, there are no nontrivial idempotents; i.e., there is a single sub-Peirce component, which is a matrix algebra $\mathrm{M}_{n}(W)$ over a local ring $W$; cf. Remark 2.33 .

We recall from Lemma 2.17 and Lemma 2.18 that, in the notation of Lemma 2.32 for $F$ infinite, $A$ is PI-equivalent to $\mathrm{M}_{n}(F)$ iff $W$ is commutative. Thus, this case reduces to the case of a single point, and the radical substitutions in $W$ are irrelevant. Thus, we must find a way of disregarding arrows connecting identically glued vertices, which we do in $\$ 5$.

4.3. Paths without gluing. Let us consider a representation whose full quiver is a path without gluing; the full quiver has the form

$$
I_{n_{1}\left(t_{1}\right)} \longrightarrow I I_{n_{2}\left(t_{2}\right)} \longrightarrow \cdots \text {. }
$$

When $t_{1}=t_{2}=\cdots=1$, this algebra appears as the Giambruno-Zaicev algebra of 12 .

In order to construct critical nonidentities of paths, it is useful to know the values of substitutions in some basic polynomials.

Example 4.10. The product $f=y_{1} \cdots y_{\ell}$ is an identity of any algebra (without unit) corresponding to the path

$$
q: \circ \rightarrow \circ \rightarrow \cdots \rightarrow \circ
$$


of length $\ell-1$. On the other hand, $y_{1} \cdots y_{\ell-1}$ is a nonidentity, which is critical for algebras over an infinite field iff $n_{1}=\cdots=n_{\ell}=1$.

Definition 4.11. Given a path $\mathcal{B}$ of length $\ell$ as in equation (12), we take indeterminates labelled $x_{i, 1}, x_{i, 2}, \ldots$, and $y_{i}$ and define

$$
f_{i}(x, y)=\left[h_{n_{i}}\left(x_{i, 1}, x_{i, 2}, \ldots\right), y_{i}\right] .
$$

We then define

$$
\Phi(\mathcal{B})=h_{n_{1}} f_{1} h_{n_{2}} f_{2} \cdots f_{\ell} h_{n_{\ell}+1},
$$

where different indeterminates are used in each polynomial, so that $\Phi(\mathcal{B})$ is multilinear.

The situation is altered a bit by empty vertices; then in equation (14) we just take

$$
f_{i}=y_{i}
$$

Remark 4.12. Any nonzero value of $\Phi(\mathcal{B})$ requires radical substitutions of the $y_{i}$.

Unfortunately, gluing can cause this polynomial to vanish. In particular, empty vertices involve extra subtleties in connection with gluing. Consider the example

$$
\left\{\left(\begin{array}{ccc}
0 & \alpha & * \\
0 & 0 & \alpha \\
0 & 0 & 0
\end{array}\right): \alpha \in F\right\} .
$$

The edges in its full quiver $\quad \circ-\alpha \rightarrow \circ-\alpha \rightarrow \circ$ are glued, so from one point of view, the vertices are also glued, although they are empty.

(1) The full quiver $I_{n_{1}(t)} \rightarrow I$ corresponds to an algebra $A$ as in Example 4.9)(3), but with identical gluing, and with $n_{1}=n_{2}$. For $n_{1}=n_{2}=1$,

$$
A=\left\{\left(\begin{array}{cc}
\alpha & \beta \\
0 & \alpha
\end{array}\right): \alpha \in F_{1}, \beta \in L\right\},
$$

where $F_{1}, L$ are as at the beginning. Note for arbitrary $n_{1}$ that $A \cong$ $\mathrm{M}_{n_{1}}\left(F[\lambda] /\left\langle\lambda^{2}\right\rangle\right)$. When $F$ is infinite, $\operatorname{id}(A)=\operatorname{id}\left(\mathrm{M}_{n_{1}}(F)\right)$, but this is never the case for $F$ finite, since for $|F|=q, A$ satisfies the identity $\left(g^{q}-g\right)^{2}$, for any central polynomial $g:=g(x)$ of $n_{1} \times n_{1}$ matrices (since $g^{q}-g$ is a nonidentity that takes values only in the radical).

The same considerations apply to paths of longer length.

Example 4.13. We construct a critical nonidentity for an arbitrary path $\mathcal{B}$ of length $\ell$, without consecutive gluing of vertices. Write $B_{i}$ for the block of the algebra $A$ of $\mathcal{B}$ corresponding to the $i$ vertex. Recall that over infinite fields, $h_{n_{i}}$ is critical central for the block $B_{i}$, and, when $F=\mathbb{F}_{q}, h_{n_{i} ; t_{i}}$ is critical central for $B_{i}$.

We see that $f_{i}(x, y)=\left[h_{n_{i}}\left(x_{i, 1}, x_{i, 2}, \ldots\right), y_{i}\right]$ can be nonzero only for substitutions of $x_{i, 1}, x_{i, 2}, \ldots$ to the same diagonal block and a radical substitution of $y_{i}$ to a block whose edge either begins or ends in this block. When the $i$-th vertex is empty we just take $f_{i}=y_{i}$. Following this argument along all the blocks shows that $f_{1} f_{2} \cdots f_{m}$ could have a nonzero evaluation iff $m$ is at most the number of edges in the path, which is $\ell$. In particular,

$$
h_{n_{1}} f_{1} h_{n_{2}} f_{2} \cdots f_{\ell+1} h_{n_{\ell+2}} \in \operatorname{id}(A) .
$$


More significantly, the polynomial $\Phi(\mathcal{B})$ is a critical nonidentity, the only possible nonzero specializations occurring in $A$ when we have the substitutions as described above and the substitutions of the $y_{r}$ are radical.

We are ready to define $\Phi$ on a path $\mathcal{B}$ without identical gluing, utilizing Example 4.13.

Remark 4.14. We write $f=h_{n_{1}} f_{1} h_{n_{2}} f_{2} \cdots f_{\ell} h_{n_{\ell+1}}$, where $\ell+1$ is the number of sub-Peirce components of $\mathcal{B}$.

(If there is no identical gluing, then $\ell$ is the length of $\mathcal{B}$.)

We take $f_{i}=\left[h_{n_{i}}\left(x_{i, 1}, x_{i, 2}, \ldots\right), y_{i}\right]$ (all $i \leq \ell$ ) unless the $i$-th vertex is empty, in which case we just take $f_{i}=y_{i}$.

4.4. Paths in which consecutive vertices are identically glued. Unfortunately, when a path $\mathcal{B}$ has glued vertices, the polynomial given in Example 4.13 might turn out to be an identity of the algebra of $\mathcal{B}$. Fortunately, this only happens when consecutive vertices are glued. The extreme case of gluing is when all vertices are glued together; i.e., in

$$
I_{n(t)} \longrightarrow I \longrightarrow \ldots \longrightarrow I \text {. }
$$

Example 4.15. The local algebra $A$ corresponding to the path

$$
I \longrightarrow I \longrightarrow I \longrightarrow \cdots
$$

of length $k$ is in the variety defined by the identity of Lie nilpotence of degree $k+1$ : $f=\left[\ldots\left[y_{1} y_{2}\right] \ldots y_{k+1}\right]$.

4.4.1. Compressed paths. By Lewin's theorem [20], when $F$ is an infinite field, isolated glued triangles do not affect the PIs, and thus we may cancel the extraneous glued arrows in the compressed full quiver in this case. But even for $F$ infinite, there is a subtle difference among the identities corresponding to certain paths, as evidenced by the following example:

Example 4.16. Although the algebras corresponding to the paths

$$
I I \longrightarrow I \longrightarrow I, \quad I I \longrightarrow I
$$

are PI-equivalent (since the first ends in an isolated glued triangle), the algebras corresponding to the paths

$$
I \longrightarrow I I \longrightarrow I \longrightarrow I, \quad I \longrightarrow I I \longrightarrow I
$$

are not PI-equivalent, since $\left[\left[x_{1},\left[y_{1,1}, y_{1,2}\right]\left[y_{2,1}, y_{2,2}\right]\right], x_{2}\right]$ is a nonidentity for the first but is an identity for the second. (The idea here is that $x_{2}$ could be specialized to an "infinitesimal", in the sense of [8, §5.2].)

Example 4.17. Let us consider the algebra without 1 corresponding to the path

$$
I \longrightarrow 0 \longrightarrow I \longrightarrow I \text {. }
$$

Explicitly, first we consider the polynomial $\left[y_{1,1}, y_{1,2}\right] x_{1}\left[y_{2,1}, y_{2,2}\right] x_{2}$. Any nonzero substitutions of $\left[y_{1,1}, y_{1,2}\right]$ and $\left[y_{2,1}, y_{2,2}\right]$ must be to the radical, above the diagonal, which means that $x_{1}$ must go to a diagonal element. In order for the polynomial $z\left[y_{1,1}, y_{1,2}\right] x_{1}\left[y_{2,1}, y_{2,2}\right] x_{2}$ not to vanish, the positioning of the empty block forces a radical substitution of $z$. Consequently, the polynomial

$$
\left[x_{3}, z\left[y_{1,1}, y_{1,2}\right] x_{1}\left[y_{2,1}, y_{2,2}\right] x_{2}\right]
$$


vanishes on this algebra because the radical components have all been exhausted in the substitutions.

In general, compression of full quivers has a secondary effect on identities. Recall from $\left[8\right.$ that $I(n)$ denotes a vertex whose base ring is the local ring $F[\epsilon] /\left\langle\epsilon^{n}\right\rangle$, where other vertices may be glued through the natural copy of $F$ in this ring.

Example 4.18. (i) Let us compare the algebra $A$ corresponding to the path

$$
I \longrightarrow I I \longrightarrow I
$$

with the quotient algebras $A_{n}$ corresponding to the paths

$$
I \longrightarrow I I \longrightarrow I(n) \text {. }
$$

Let $\operatorname{id}\left(A_{n}\right)$ denote the corresponding identities. Then $\operatorname{id}(A)=\bigcap \operatorname{id}\left(A_{n}\right)$, since $F[\lambda]$ can be embedded into the ultraproduct of the $F[\lambda] /\left\langle\lambda^{n}\right\rangle$.

Similarly to (i), compare the algebra $A^{\prime}$ corresponding to

$$
I \longrightarrow I I
$$

with its series of quotient algebras $A_{n}^{\prime}$ corresponding to

$$
I \longrightarrow I(n) \text {. }
$$

Again, $\operatorname{id}\left(A^{\prime}\right)=\bigcap \operatorname{id}\left(A_{n}^{\prime}\right)$.

These exemplify the following phenomenon.

Remark 4.19 (Compression and gluing). When $F$ is infinite, any commutative $F$ algebra with 1 satisfies the same identities as $F$, since extending an infinite base field does not affect PIs; thus, compression does not change the corresponding variety when $F$ is infinite.

Nevertheless, for $F$ finite, letting $\mathcal{I}_{k}$ denote the identities corresponding to the full quiver having compression of degree $k$, and $\mathcal{I}_{\infty}$ denote the identities corresponding to the full quiver having no compression, we see that $\mathcal{I}_{j} \subsetneq \mathcal{I}_{k}$ for $j>k$, and $\mathcal{I}_{\infty}=\bigcap_{k \in \mathbb{N}} \mathcal{I}_{k}$.

4.4.2. Examples of gluing up to infinitesimals. In [8, Example 5.3] we provided some examples of gluing up to infinitesimals. Even in a path, gluing up to infinitesimals can provide unexpected identifications.

Example 4.20. Consider the paths $I \rightarrow I \rightarrow I I \rightarrow I$ and $I \rightarrow I I \rightarrow I \rightarrow I$. In [8, Example 5.4(viii)] it is shown that the corresponding algebras are generated by $Y_{i}=\left(\begin{array}{ccc}\xi_{i 1} & * & * \\ 0 & \xi_{i 2} & * \\ 0 & 0 & \xi_{i 1}+\varepsilon_{i}\end{array}\right)$, where the $\varepsilon_{i}$ are "infinitesimal elements of second order" and $*$ denotes arbitrary entries.

This example also is symmetric, in the sense that the matrices $Y$ could be rewritten as

$$
\left(\begin{array}{ccc}
\xi_{i 3}-\varepsilon_{i} & * & * \\
0 & \xi_{i 2} & * \\
0 & 0 & \xi_{i 3}
\end{array}\right)
$$

where $\xi_{i 3}=\xi_{i 1}+\varepsilon_{i}$, so one could reverse the roles of $\xi_{i 1}$ and $\xi_{i 3}$.

Hence, the two algebras are PI-equivalent. This proves the nontrivial statement that these two different paths correspond to the same variety, and thus our map $\Phi$ does not provide a well-defined map on relatively free PI-algebras of paths, although 
we do not know of a graph-theoretic demonstration of this fact. This example should be contrasted with Example 4.16.

Since consecutive gluing of edges may introduce new commutative rings, there is a big difference between the finite and infinite cases. When $F$ is finite, one can distinguish among the identities.

Example 4.21. Explicitly, $\mathbb{F}_{q}[\lambda] /\left\langle\lambda^{\ell}\right\rangle$ satisfies the identity $\left(x^{q}-x\right)^{\ell}$, when $q<\infty$, as well as all the identities of $\mathbb{Q}$. Thus, we can distinguish among the glued triangles over different finite fields, by means of polynomial identities.

Example 4.22. Let $F$ be the field $\mathbb{F}_{16}$ of order 16 , and $C=\mathbb{F}_{4}\left[\varepsilon_{2}:\left(\varepsilon_{2}\right)^{2}=0\right]$, also a commutative ring of order 16 . $F$ satisfies the identity $\lambda^{16}-\lambda$. On the other hand, since the radical of $C$ is $\left\langle\varepsilon_{2}\right\rangle$, we see that $C$ satisfies the identity $\left(\lambda^{4}-\lambda\right)^{2}=\lambda^{8}-\lambda^{2}$.

Next, let us consider a strange effect when some, but not all, of the edges are glued.

Example 4.23. The identities of the path

$$
I \longrightarrow \alpha \longrightarrow I \longrightarrow \alpha \longrightarrow I \longrightarrow \alpha \longrightarrow \cdots-\alpha \longrightarrow I
$$

are a consequence of the Lie nilpotence identity of degree $k+1$ and the metabelian identity $[[x, y],[z, t]]$. In this case we can represent the algebra by $2 \times 2$ matrices over the ring $F[t] /\left\langle t^{k+1}\right\rangle$.

4.5. Frobenius gluing. The analogous considerations hold when the vertices have Frobenius gluing. Recall that Frobenius gluing only arises when $F$ is finite. If there is Frobenius gluing, then we use $q$-skewed commutators, defined as follows.

Definition 4.24. Define $[a, b]_{q}=a b-b a^{q},{ }_{q}[a, b]=a^{q} b-b a$, and $[a, b, c]_{q}=$ $a b c-c b a^{q}$. Also, define $\operatorname{ad}_{q}(a)$ by $\operatorname{ad}_{q}(a)(b)=[a, b]_{q}$.

When Frobenius gluing is coupled with gluing of edges (which necessitates gluing of the corresponding vertices), the critical polynomials may involve repeated skewed commutators.

Example 4.25. The full quiver $I_{n_{1}(t)} \rightarrow I^{(\ell)}$ corresponds to the algebra $A$ as in Example 4.9(3), but with Frobenius gluing. For $n_{1}=n_{2}=1$ we have

$$
A=\left\{\left(\begin{array}{cc}
\alpha & \beta \\
0 & \alpha^{q^{\ell}}
\end{array}\right): \alpha \in F_{1}, \beta \in L\right\},
$$

where $F_{1}, L$ are as in $\$ 4.2$. Now we have the identity $g^{q^{\ell}} y-y g$, for any central polynomial $g$ of $n_{1} \times n_{1}$ matrices.

Example 4.26. (i) $I^{(1)} \longrightarrow I I \longrightarrow I$, with $q=2$; the Frobenius gluing is by squaring. Now we have the identity $\left[x_{1},\left[y_{1,1}, y_{1,2}\right] x_{2}\left[y_{2,1}, y_{2,2}\right], x_{3}\right]_{2}$.

Example 4.27. Take the algebra $A(k)$ corresponding to the path

$$
I^{(1)} \longrightarrow I I \longrightarrow I(k) \text {. }
$$

Consider the polynomial

$$
f=\operatorname{ad}_{q}\left(x_{3}\right)^{s}\left(\left[x_{1}, y_{1}\right] x_{1}\left[x_{2}, y_{2}\right] x_{2}\right) ;
$$


$f \in \operatorname{id}(A)$ iff $s>[(k-1) / q]$. For $s=[(k-1) / q]$, any evaluation lands in the maximum power of the radical. Moreover $A\left(k_{1}\right)$ is PI-equivalent to $A\left(k_{2}\right)$ iff $\left[\left(k_{1}-\right.\right.$ $1) / q]=\left[\left(k_{2}-1\right) / q\right]$.

Remark 4.28. There is a basic difference between identical and nonidentical Frobenius gluing, which is reflected in the identities. (Nonidentical) Frobenius gluing is automatically removed upon tensoring up to the algebraic closure $\bar{F}$ (or, in fact, by any infinite field).

We recall part of 8 :

Example 4.29. (i) Suppose $A$ is the algebra of a path of length $\ell$ with nonidentical Frobenius gluing, with each $n_{i}=1$. Then the algebra $A \otimes \bar{F}$ is isomorphic to the algebra $T$ of upper triangular $\ell \times \ell$ matrices over $\bar{F}$, which corresponds to the path $I \rightarrow I I \rightarrow I I I \rightarrow \cdots$.

(ii) On the other hand, the conclusion of (i) fails if $A$ has identical gluing or Frobenius gluing up to infinitesimals, and in fact $\operatorname{id}(A \otimes \bar{F}) \supset \operatorname{id}(A)$. In this case, we obtain two different varieties which satisfy the same multilinear identities and thus have the same codimension growth.

[8. Example 5.2] provides an example of a commutative algebra with nonFrobenius gluing on radical components.

\section{Trace-ABsorbing NONIDEntities And Their T-IDEALS}

We get to the main point of this paper, namely to show that any relatively free representable algebra $A$ has a T-ideal in common with a Noetherian algebra $A^{\prime}$ properly containing $A$ (which is a finite module over a commutative affine algebra when $A$ is affine). This T-ideal corresponds to a critical nonidentity $\Phi(\mathcal{B})$ of a branch $\mathcal{B}$ of $A$.

The method is to define some action of characteristic coefficients (i.e., coefficients of the characteristic polynomial) of elements of a Zariski-closed algebra $A$, such that the values of the nonidentity obtained from its full quiver are closed under multiplication by these characteristic coefficients. This will enable us to preserve Hamilton-Cayley type properties in the evaluations of diagonal blocks, and plays a key role in combinatorial PI-theory because of Shirshov's Theorem [6, Chapter 2].

The only technique that we have so far to incorporate multiplication by characteristic coefficients into evaluations of polynomials is Remark 2.16. which only works for semisimple elements.

Ironically, over a field of characteristic $p$, this is enough, by [6, Remark 2.35 and Lemma 2.36], which we quote:

Lemma 5.1. When $p^{m}>n$ (which is greater than the nilpotence index of the Jacobson radical), then the element $a^{p^{m}}$ is semisimple for every $a \in A$.

So in the polynomial $f$ we just replace each $x_{i}$ by $x_{i}^{p^{m}}$. Thus, when studying algebras over fields, we need only worry about characteristic 0 , in which case all characteristic coefficients can be described in terms of traces. Furthermore, since we cannot have a nontrivial Frobenius twist, $\$ 4.5$ is irrelevant to this section.

Remark 5.2. We obtain the desired trace action in three steps:

(1) Construct a polynomial $f$ via a branch of the full quiver of $A$, which will take on a nonzero value for substitutions in a path to radical elements 
corresponding to the arrows of the path joining different Peirce components. (Some of them could correspond to empty vertices.) This was done in Remark 4.14, based on the polynomial of Definition 4.11, in view of the argument of Example 4.13 .

(2) A procedure of further specializing the polynomial so that we can bypass radical substitutions of arrows connecting glued vertices. This will be done by "hiking" the polynomial, in Lemma 5.8. In this case the sub-Peirce components coincide with the Peirce components of $A$, thereby simplifying our discussion; hiking also ensures that the matrix traces in any Peirce component of $A$ commute. (When studying algebras over a field we can reduce our use of characteristic polynomials to traces, in view of Lemma 5.1)

(3) Continuing the previous step, we need an action of matrix traces (computed on the diagonal components of the given representation of $A$ ) on the Tideal of $f$. To do this, one computes the trace as in Definition 2.25, and applies this on each (glued) matrix component, i.e., the Peirce components corresponding to vertices on each side of an arrow. More precisely, suppose we have two Peirce components, whose idempotents are $e_{r}=\sum_{k} e_{r(k)}$ and $e_{s}=\sum_{\ell} e_{s(\ell)}$. For any arrow $\alpha$ from (nonglued) vertices $r_{k}$ to $s_{\ell}$, we consider the matrix $\left(a_{u v}\right)$ corresponding to $\alpha$, and take traces on the $r_{k}$-diagonal component on the left, and the $s_{\ell}$-diagonal component on the right. In other words, if the vertex corresponding to $r$ has matrix degree $n_{r}$, taking an $n_{r} \times n_{r}$ matrix $w$, we define $\operatorname{tr}_{u}(w)$ as in the action of Remark 2.1 and then the left action

$$
a_{u, v} \mapsto \operatorname{tr}_{u}(w) a_{u, v}
$$

and likewise for an $n_{s} \times n_{s}$ matrix $w$ we define the right action

$$
a_{u, v} \mapsto a_{u, v} \operatorname{tr}_{v}(w)
$$

(However, we only need the action when the vertex is nonempty; we forego the action for empty vertices.)

Unfortunately the product of an element of $A$ with $\operatorname{tr}(a)$ need not lie in $A$. Thus, we turn to the alternative action of the trace given in Remark 2.26. Equation (44) of Remark 2.26 then shows that left multiplication by $\operatorname{tr}(a)$ acts on the set of evaluations of any $n_{i}^{2}$-alternating polynomial $f(x ; y)$ on an $n_{i} \times n_{i}$ matrix component. By the previous step, these two trace actions coincide on the T-ideal of $f$. In the presence of gluing, one needs a more sophisticated argument for substitutions in radical components, using hiking, as is explained in Theorem 5.14.

In order to implement this program, we need a new notion.

Definition 5.3. Given a quasi-linear $F$-homogeneous polynomial $f(x ; y ; z)$ in indeterminates labelled $x_{i}, y_{i}$, we say $f$ is trace-absorbing with respect to a full quiver $\Gamma=\Gamma(A)$ if the following properties hold:

(1) $f$ specializes to 0 under any substitution in which at least one of the $x_{i}$ is specialized to a radical element of $A$. (In other words, the only possible nonzero values of $f$ are obtained when all substitutions of the $x_{i}$ are semisimple.) 
(2) $f(\mathcal{A}(\Gamma))^{+}$is closed under multiplication (via specializations of the $x_{i}$ ) by any characteristic coefficient of any element in a simple (diagonal) matrix block of $\mathcal{A}(\Gamma)$.

Example 5.4 (Trace-absorbing nonidentities for elementary full quivers). In Example 4.9, $x$ is a trace-absorbing nonidentity for the full quiver $\circ \rightarrow \circ$.

The polynomial $\left[x_{1}, x_{2}\right] x_{3}$ is a trace-absorbing nonidentity for the full quiver $\circ \rightarrow \bullet$ for $n_{1}=n_{2}=1$ since we have the substitution

$$
\left[e_{12}, e_{22}\right] e_{22}=e_{12} \neq 0 \text {. }
$$

When $|F|=p^{t}$, this nonidentity is not critical. To make it critical we should take $[x, y]\left(z^{p^{t}}-z\right)$.

More generally, the full quiver $\circ \rightarrow I_{n(t)}$ has the trace-absorbing nonidentity $\left[x, h_{n}\right] c_{n^{2}}$.

Similarly, the polynomial $c_{n^{2}}\left[x, h_{n}\right]$ is a trace-absorbing nonidentity for the full quiver $I_{n(t)} \rightarrow \circ$, and the polynomial $c_{n_{1}^{2}}\left[x, h_{n}\right] c_{n_{2}^{2}}$ is a trace-absorbing nonidentity for the full quiver $I_{n_{1}\left(t_{1}\right)} \rightarrow I I_{n_{2}\left(t_{2}\right)}$.

Lemma 5.5. In each instance of Example 5.4, the only nonzero evaluations of the given trace-absorbing nonidentity are in the radical.

Proof. The commutator involving the central polynomial yields 0 in the diagonal block, and thus must be in the radical component.

Remark 5.6. Our main objective is to introduce trace-absorbing polynomials corresponding to all canonical full quivers. By Lemma [5.1] we may assume that all characteristic coefficients can be described in terms of traces. As noted in Step (3) above, we have the natural bimodule action of traces on $A$ given in terms of the full quiver, which we can identify with $\operatorname{tr}_{\text {pol }}(a)$, defined in equation (4), whenever the matrix traces commute. (In particular, this technique works for a single diagonal matrix block over a commutative ring.)

To treat the general case, we need another idea to force the matrix traces to commute with each other and also with radical substitutions of arrows connecting glued vertices. The following well-known observation is needed.

Remark 5.7. If $f\left(x_{1}, \ldots, x_{n}\right)$ is any multilinear polynomial, then

$$
f\left(a_{1}, \ldots, a_{n}\right) a-a f\left(a_{1}, \ldots, a_{n}\right)=\sum_{i} f\left(a_{1}, \ldots,\left[a_{i}, a\right], \ldots, a_{n}\right) .
$$

Lemma 5.8. Suppose a quasi-linear nonidentity $f$ of a Zariski closed algebra A has a nonzero value for some semisimple substitution of some $x_{i}$ in $A$, corresponding to a vertex in the full quiver labelled by $n_{i}\left(t_{i}\right)$. Taking the maximal such $n_{i}$ and replacing $x_{i}$ by $\left[x_{i}, h_{n_{i}}\right]$ (where $h_{n_{i}}$ involves new indeterminates) yields a quasilinear polynomial

$$
\nabla_{i} f:=f\left(\ldots,\left[x_{i}, h_{n_{i}}\right], \ldots\right)
$$

in which any nonzero substitution forces us into a higher power of the radical $J$ of A.

Proof. Each commutator takes its evaluation in the radical, since the evaluations of $h_{n_{i}}$ in the semisimple part are central. This forces a radical substitution of $\left[x_{i}, h_{n_{i}}\right]$ to replace the original semisimple substitution of $x_{i}$. 
Moreover, $\nabla_{i} f$ is quasi-linear since the expression $\left[x_{i}, h_{n_{i}}\right]$ is linear in each indeterminate.

We call this procedure (application of (27)) hiking the polynomial $f$. We say such a nonidentity $f$ is fully hiked if it becomes an identity after any further hike. Recall that the radical of a Zariski-closed algebra $A$ is nilpotent.

Proposition 5.9. For any Zariski-closed algebra $A$ of nilpotence index $k$, any polynomial as in Lemma 5.8 can be hiked at most $k-1$ times to get a fully hiked nonidentity.

Proof. Since the product of any $k$ elements of the radical is 0 by definition, we can only hike $f$ at most $k$ times before getting an identity.

Remark 5.10. Note that hiking absorbs all radical substitutions joining sub-Peirce components of the same Peirce component, since we can apply these substitutions in evaluating the $h_{n_{i}}$ in equation (27), so when working with fully hiked polynomials, we need consider only the Peirce decomposition (and not the more complicated sub-Peirce decomposition).

Example 5.11. This technique is illustrated via the full quiver given for the Grassmann algebra:

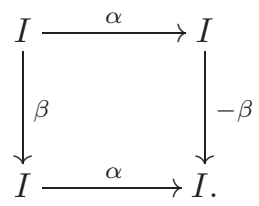

Clearly the critical nonidentity for each branch is $\left[x_{1}, x_{2}\right]$, and we get the Grassmann identity $\left[\left[x_{1}, x_{2}\right], x_{3}\right]$ by taking $f=x_{1}$ and applying Proposition [5.9] The best way to understand the PIs is to include in the full quiver the diagonal edges in each square face of the cube. Indeed, one could draw two diagonals (corresponding to the two choices of $\alpha$ and $\beta$ ), but these coincide in $\left[x_{1}, x_{2}\right]$, because of the factor of -1 in the proportional gluing.

Example 5.12. We modify each $f_{i}$ in Remark 4.14 via hiking, in case the corresponding Peirce component $A_{i} \cong \mathrm{M}_{n_{i}}\left(W_{i}\right)$. Note that $W_{i}$ might intersect the radical of $A$, according to the number of glued vertices, so we need to account for arrows yielding radical substitutions in $W_{i}$, by this hiking procedure. For the first two consecutive glued vertices of $A_{i}$, we start with

$$
f_{i}=y_{i}
$$

(since the diagonal substitutions in $A_{i}$ are scalar, and thus central). If the portion of $\mathcal{B}$ corresponding to $A_{i}$ contains another glued vertex, we hike by replacing $y_{i}$ by the Lie commutator $\left[y_{i}, z_{i, k}\right]$, for a new indeterminate $z_{i, k}$, in order to force two radical substitutions of the $y_{i}$ as long as we encounter glued vertices in $A_{i}$. (For empty vertices we apply (15) to the process.) Thus, if $A_{i}$ has $t_{i}$ glued vertices, we replace $y_{i}$ by the higher Lie commutator

$$
\left[\left[\ldots\left[\left[y_{i}, z_{i, t_{i}}\right], z_{i, t_{i}-1}\right], \ldots\right], z_{i, 1}\right] .
$$


Remark 5.13. Suppose $x_{i}$ corresponds to an arrow joining vertices of matrix degree $n_{i}$ and $n_{i}^{\prime}$. As explained in [6, p. 27], there is a polynomial $\tilde{c}_{n_{i}^{2}}$ such that

$$
\tilde{c}_{n_{i}^{2}}(y) x_{i} c_{n_{i}^{\prime 2}}(y)=\operatorname{tr}\left(y_{1}\right) c_{n_{i}^{2}}(y) x_{i} c_{n_{i}^{\prime 2}}(y)
$$

on any diagonal block. Since traces commute on any diagonal block, we conclude from this that

$$
\tilde{c}_{n_{i}^{2}}(y) x_{i} c_{n_{i}^{\prime 2}(y)} \tilde{c}_{n_{i}^{2}}(z) x_{i} c_{n_{i}^{\prime 2}(z)}-\tilde{c}_{n_{i}^{2}}(z) x_{i} c_{n_{i}^{\prime 2}(z)} \tilde{c}_{n_{i}^{2}}(y) x_{i} c_{n_{i}^{\prime 2}(y)}
$$

vanishes identically on any diagonal block. One concludes from this that substituting (30) for $x_{i}$ hikes our polynomial one step further.

Recall that the T-space of a polynomial $f$ on an algebra $A$ is defined as the subspace of $A$ spanned by the evaluations of $f$ on $A$. The following result holds for arbitrary algebras of paths.

Theorem 5.14. Any path has a trace-absorbing nonidentity. More precisely, any fully hiked polynomial $f$ obtained from hiking the polynomial of Remark 4.14 is trace-absorbing. In fact, the T-spaces evaluated on $A=\mathcal{A}(\Gamma)$ of our critical nonidentities of $A$ are closed under multiplication by traces (and more generally by all the characteristic coefficients) of the simple components of semisimple elements.

Proof. Since by definition any further hike of $f$ yields an identity, Remark 5.13 dictates that polynomial traces defined in terms of $f$ via equation (4) must commute. There is a subtlety involved when the Peirce component involves consecutively glued vertices, since then we need the radical substitution $b$ of an arrow connecting glued vertices to commute with $\operatorname{tr}(a)$. But this is seen via Remark 5.7 since the right side of (26) hikes the polynomial further and thus is 0 . Thus, the polynomial action on $\mathcal{I}$ coincides with the well-defined matrix action as described in Remark 5.2(3).

Since the critical nonidentities all contain an $n^{2}$-alternating polynomial at each component, and we apply the trace action simultaneously to each of these polynomials, their T-spaces are closed under multiplication by characteristic coefficients of the simple components of semisimple elements, so we have a trace-absorbing polynomial.

We have completed the first step in our program:

Definition 5.15. Formally, we define $\Phi(\mathcal{B})$ to be the fully hiked, trace-absorbing polynomial we have obtained in Theorem 5.14.

Theorem 5.16 (Trace Adjunction Theorem). For any branch $\mathcal{B}$ of a basic full quiver of a representable relatively free algebra $A$, the image in $A$ of the T-ideal $\mathcal{I}$ of the polynomial $\Phi(\mathcal{B})$ is also an ideal of the algebra $\hat{A}$ obtained by adjoining to $A$ the matrix traces of the Peirce components of the generic generators of $A$.

Proof. In view of [7, Theorem 7.20 and Corollary 7.21], we may assume that $A$ is affine, and its generators are generic elements, say $X_{1}, \ldots, X_{t}$. We adjoin the Peirce components of these generic elements, noting that because the polynomial $\Phi(\mathcal{B})$ obtained by Theorem 5.14 is fully hiked, all substitutions involving these Peirce components are a product of a maximal number of radical elements and thus still are in its T-ideal $\mathcal{I}$. But now we also adjoin the matrix traces for all the Peirce components (as described by equations (24) and (25) in Remark $5.2(3)$ ) of these new components (applied simultaneously to each generator and each Peirce component) 
to obtain the algebra $\hat{A}$. As before, Remark 5.13 dictates that polynomial traces defined in terms of $f$ via equation (4) must commute, and applying Remark 5.7 shows that they commute with any radical substitutions of arrows connecting glued vertices. Thus, the action coincides with the matrix action defined on $\mathcal{I}$.

Example 5.17. (1) Consider the algebra corresponding to the path

$$
\mathcal{B}:=\quad I \longrightarrow I \longrightarrow I \longrightarrow I I \longrightarrow I \longrightarrow I I,
$$

represented in $6 \times 6$ matrices. Note that the first three vertices are glued, and we have the Peirce idempotents $e_{1}=e_{11}+e_{22}+e_{33}+e_{55}$ and $e_{2}=$ $e_{44}+e_{66}$. The trace actions of $w$ on the components $a_{u, v}$ are just given by left and right multiplication by $w$ viewed as a scalar. The corresponding polynomial is $\Phi(\mathcal{B})=x_{1}\left[y_{1}, y_{2}\right] x_{2}\left[x_{3}, y_{3}\right] x_{4}\left[x_{5}, y_{5}\right] x_{6}$, which is the same as for

$$
\mathcal{B}^{+}:=\quad I \longrightarrow I \longrightarrow I \longrightarrow I I \longrightarrow I I I \longrightarrow I I .
$$

(2) Consider the algebra without 1 corresponding to the path

$$
\mathcal{B}^{\prime}:=\quad I \longrightarrow I \longrightarrow I \longrightarrow I I \longrightarrow \circ \longrightarrow I I I,
$$

represented in $6 \times 6$ matrices. Since the first three vertices are glued, we have the Peirce idempotents $e_{1}=e_{11}+e_{22}+e_{33}, e_{2}=e_{44}$, and $e_{3}=e_{66}$.

$\Phi\left(\mathcal{B}^{\prime}\right)$ starts with $x_{1}\left[y_{1}, y_{2}\right] x_{2}$ and then continues with $\left[x_{3}, y_{3}\right]$ and the empty vertex and then $\left[x_{4}, y_{4}\right] x_{5}$, yielding altogether the polynomial

$$
x_{1}\left[y_{1}, y_{2}\right] x_{2}\left[x_{3}, y_{3}\right]\left[x_{4}, y_{4}\right] x_{5} .
$$

Note here there is ambiguity as to whether $x_{3}$ or $y_{3}$ gets a radical substitution, and likewise for $x_{4}$ and $y_{4}$. (Here the traces are just the scalars, since all matrices have degree 1.) We can view $\left[y_{1}, y_{2}\right]$ as a "hiking" of $y$ which corresponds to the radical of the first Peirce component, a local algebra.

Remark 5.18. In view of Theorem 5.16, when studying representable relatively free algebras, we may assume that they are affine, in which case the algebra $\hat{A}$ of Theorem 5.16 is a finite module over a commutative affine algebra, by Shirshov's Theorem [6, Chapter 2].

However, this may not be possible when the algebra is not representable, as in the case of the infinite dimensional Grassmann algebra.

Theorem 5.19. For any full quiver $\Gamma$, there is a trace-absorbing nonidentity for the algebra of $\Gamma$.

Proof. We obtained this trace-absorbing nonidentity for paths in Theorem 5.14. For the general case, we apply $\Phi$ to a maximal branch (i.e., first, with respect to the maximal size of the matrix components, and then with respect to the maximal length). (If there are several branches of the same dimension vector, we pick one arbitrarily.)

Note 5.20. When working in nonzero characteristic, we need to treat all the characteristic coefficients, and not just the traces. This is discussed in detail in our next paper [10]. 


\section{Rationality of Hilbert series}

Our next application concerns the theorem that the Hilbert series of a representable relatively free affine PI-algebra $A$ is rational.

A proof is given in [6, Theorem 9.44], but it relies on the full force of Kemer's theory, in particular Kemer's solution of Specht's Problem in Characteristic 0. Our aim is to indicate how the proof in [6. Theorem 9.44] can be streamlined by means of Theorem 5.14, full details are given in 9 .

6. Lemma 9.40(i)] says that if $A$ has an ideal $I$ such that $A / I$ has a rational Hilbert series and $I$ has a rational Hilbert series as an $A$-module, then $A$ has a rational Hilbert series.

In 6] such an ideal $I$ is obtained by means of Noetherian induction, which is available since Kemer's solution of Specht's Problem implies the ACC on T-ideals. However, it is much easier to find such an ideal $I$ by means of the full quiver of $A$. First we take any branch of maximal length of $\Gamma$, and its associated trace-absorbing polynomial. Then the T-ideal is a common ideal of $A$ and $\hat{A}$ (of Remark 5.16) which is a finite module over a commutative affine algebra. Hence any such T-ideal $I$ is well known to have a rational Hilbert series as an $A$-module. Since this argument applies to all such ideals $I$, all of which are ideals in common with $\hat{A}$, we can mod out by their sum, and thereby have eliminated all the branches of $\Gamma$. The image of $A$ is thus a subalgebra of the image of $\hat{A}$, which is representable having Jacobson radical of lower nilpotence index, so by induction is representable. Thus, we conclude by induction.

\section{Parametric SETS OF IDEntities}

Having obtained trace-absorbing nonidentities in \$5, culminating in Theorem 5.19] we change direction for the remainder of this paper and turn to identities. Here is an example of a path giving rise to a subtle phenomenon in PI-theory.

Definition 7.1. A parametric identity of an algebra $A$ is a polynomial $f\left(x_{1}, \ldots\right.$, $\left.x_{d}\right) \in K[\Lambda]$, for some purely transcendental field extension $K=F\left(\xi_{1}, \ldots, \xi_{m}\right)$ of the base field $F$ whose homogeneous components (in the $x_{i}$ ) are not identities, but which becomes an identity for any specialization of the $\xi_{i}$ to $F$.

The theory of full quivers confronts us with the following sort of unexpected example:

Example 7.2. We give an example of an algebra $A$ without unit element (over a base field $F$ of arbitrary characteristic), whose full quiver is a path, and with a parametric polynomial identity $f$ defined over the field $K=F\left(\xi_{1}, \ldots, \xi_{2 n}\right)$, where the $\xi_{i}$ are purely transcendental over $F$.

Consider the following full quiver of length $2 n$ edges:

$$
\Gamma: \circ \longrightarrow \circ \longrightarrow \cdots \longrightarrow 0 \text {. }
$$

All diagonal blocks are zero-dimensional, so the corresponding algebra $A$ (without 1 ) is nilpotent of index $2 n+1$, in particular satisfying the identity $x^{2 n+1}$.

The edges of $\Gamma$ are given the following extra relations:

$$
v_{i}=v_{0}+\xi_{i} v_{1}
$$


i.e., $v_{i}(a)=v_{0}(a)+\xi_{i} v_{1}(a)$ for every $a \in A$. Here $v_{i}(a), a \in A$, denote the coordinates of $a$ in the entry corresponding to the $i$-th edge of the full quiver $\Gamma$. We shall find a parametric set of identities of degree $2 n$ (of degree $n$ in both $a$ and b).

Consider $M=$ \{strings of all monomials in $a$ and $b$, of degree $n$ in each of $a$ and $b\} \subset A$. We claim that $M$ is linearly independent over $F$, but linearly dependent over $K$.

Indeed, write $x_{1}=v_{0}(a), x_{2}=v_{0}(b), y_{1}=v_{1}(a)$ and $y_{2}=v_{1}(b)$. Given a string $w \in M$, we let $\mathcal{I}_{w}$ denote the set of positions in which the letter $a$ occurs. Thus we put

$$
c_{w}=\prod_{i=1}^{2 n}\left(x_{j_{i}}+\xi_{i} y_{j_{i}}\right),
$$

where $j_{i}=1$ for $i \in \mathcal{I}_{w}$ and $j_{i}=2$ for $i \notin \mathcal{I}_{w}$, and

$$
w=c_{w} e_{1,2 n+1} .
$$

Expanding the product, it is easy to see that the coefficients of $y_{1}^{n} x_{2}^{n}$ are the products of $\xi_{i}$ for $i \in \mathcal{I}_{w}$; hence the coefficients of such monomials are linearly independent over $F$, as desired.

It follows that $A$ has no two-variable polynomial identity with coefficients in $F$ of degree $n$ with respect to each of the indeterminates.

Now we show that $A$ does have identities of such type with coefficients in the larger field $K$, for $n$ sufficiently large. The number of strings in $M$ is clearly $\left(\begin{array}{c}2 n \\ n\end{array}\right)$. The $K$-growth of $K\left[x_{1}, x_{2}, y_{1}, y_{2}\right]$ is polynomial, with Gel'fand-Kirillov dimension 4. The space spanned by strings viewed as monomials of degree $n$ in $x_{1}, y_{1}$ and degree $n$ in $x_{2}, y_{2}$ is of dimension $(n+1)^{2}$. But, for large $n,\left(\begin{array}{c}2 n \\ n\end{array}\right)>(n+1)^{2}$; hence we have a linear relation among the strings in $M$, and thus a polynomial identity with coefficients in $K$ (which are not all in $F$ ). We conclude that we have a parametric set of identities.

The same argument, using an infinite set of indeterminates, enables us to produce a PI-algebra $A$ without any 2 -variable identities of degree $\leq 2 n$ defined over $F$, but which has parametric 2 -variable identities of degree $2 n$ defined over $K$. A similar construction can also be done for a branch with a sequence of glued vertices of any dimension, but is technically more complicated.

Having produced an example of a 2 -variable PI defined over $K$ which is not a consequence of 2-variable PIs defined over $F$, we raise the following question:

Question 7.3. Given a variety $V$ of PI-algebras over a field $F$, what is the minimal subfield $F_{0}$ which contains a finite subset $S \subseteq \mathrm{id}(V)$ that generates $\mathrm{id}(V)$ as a Tideal?

\section{IDENTITIES ARISING FROM FULL QUIVERS}

Having seen that full quivers produce interesting identities, we turn to explore different identities that arise naturally from full quivers.

8.1. Identities of paths. Here are some well-known identities for paths. 
Example 8.1. Suppose $A$ is the algebra of the full quiver which is the path

$$
I_{n_{1}} \longrightarrow I I_{n_{2}} \longrightarrow I I I_{n_{3}} \longrightarrow \cdots
$$

of length $\ell-1$. In this case, $\operatorname{id}(A)=\operatorname{id}\left(\mathrm{M}_{n_{1}}(F)\right) \operatorname{id}\left(\mathrm{M}_{n_{2}}(F)\right) \cdots$ by Lewin's theorem.

(i) Let $n=\max \left\{n_{1}, \ldots, n_{\ell}\right\}$. It follows from the previous paragraph that the product of standard polynomials $s_{2 n_{1}} s_{2 n_{2}} \cdots s_{2 n_{\ell}}$ is the minimal identity of $A$, where we substitute distinct indeterminates in each standard polynomial.

For example, the full quiver

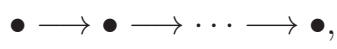

a single path of length $n=\ell$ where each $n_{i}=1$, corresponds to the algebra of upper triangular $n \times n$ matrices under the natural representation, and satisfies the identity

$$
\left[x_{1}, x_{2}\right] \cdots\left[x_{2 n-1}, x_{2 n}\right] .
$$

(ii) One could use any identity of $n_{i} \times n_{i}$ matrices in (i), instead of the standard polynomial. In particular, using the Capelli polynomial $c_{n}$, we have the identity

$$
c_{n_{1}^{2}+1} c_{n_{2}^{2}+1} \cdots c_{n_{\ell}^{2}+1},
$$

where again we use distinct indeterminates for each occurrence of the Capelli polynomial. However, $c_{n_{1}^{2}} c_{n_{2}^{2}+1} \cdots c_{n_{\ell}^{2}+1} \notin \operatorname{id}(A)$, and is a critical nonidentity for $F$ infinite.

Having provided various critical polynomials, we turn now to one which, although not necessarily of lowest degree, seems to best preserve the information contained in the full quiver. We fix the following notation for our examples.

(The identity of Example 8.1(ii) does not reflect all the information provided by the path, and certainly does not reflect the size of the base field. To treat the case of finite fields $\mathbb{F}_{q^{m}}$, we need to modify our polynomials along the lines of Proposition 4.7 or Remark 4.8)

8.2. Paths in which nonconsecutive vertices are glued. Let us consider the case for which some nonconsecutive vertices are glued. It is convenient to introduce the notation $\{a, b, c\}$ for $a b c-c b a$. We call this a transposed difference. (This is reminiscent of the Lie triple product.)

Example 8.2. (i) $I \longrightarrow I I \longrightarrow I$, with identical gluing. The corresponding algebra is

$$
\left\{\left(\begin{array}{ccc}
\alpha & * & * \\
0 & \beta & * \\
0 & 0 & \alpha
\end{array}\right): \alpha, \beta \in F\right\} \subset \mathrm{M}_{3}(F) .
$$

We consider the nonidentity $x_{1}\left[y_{1,1}, y_{1,2}\right] x_{2}\left[y_{2,1}, y_{2,2}\right] x_{3}$, which arises in Definition 4.11. In order not to get 0 at once, $x_{1}$ and $x_{3}$ must both be specialized to the glued block corresponding to I, so

$$
\begin{aligned}
\left\{x_{1},\left[y_{1,1}, y_{1,2}\right] x_{2}\left[y_{2,1}, y_{2,2}\right], x_{3}\right\}= & x_{1}\left[y_{1,1}, y_{1,2}\right] x_{2}\left[y_{2,1}, y_{2,2}\right] x_{3} \\
& -x_{3}\left[y_{1,1}, y_{1,2}\right] x_{2}\left[y_{2,1}, y_{2,2}\right] x_{1}
\end{aligned}
$$

is also an identity, which is critical over an infinite field. (Note that $x_{4}$ was superfluous in this example, since there was no gluing involved in the second component.) We can lower the degree of our identities as follows: For the full quiver 
$I \longrightarrow I I \longrightarrow I I I$ without gluing, we would have the trace-absorbing nonidentities

$$
x_{1}\left[y_{1,1}, y_{1,2}\right]\left[y_{2,1}, y_{2,2}\right], \quad\left[y_{1,1}, y_{1,2}\right]\left[y_{2,1}, y_{2,2}\right] x_{1}
$$

(and $x_{1}$ is superfluous, since it could be specialized to 1 ).

These nonidentities are not so useful in detecting the presence of gluing, since we do not know which substitution (of $y_{2,1}$ or $y_{2,2}$ ) is semisimple, to the diagonal block corresponding to I, and which substitution is radical. However, their difference

$$
\left[x_{1},\left[y_{1,1}, y_{1,2}\right]\left[y_{2,1}, y_{2,2}\right]\right]
$$

is an identity.

(ii) $I \longrightarrow I I \longrightarrow I I I \longrightarrow I I \longrightarrow I$, where each gluing is identical.

Without gluing we would have the critical nonidentity

$$
x_{1}\left[y_{1,1}, y_{1,2}\right] x_{2}\left[y_{2,1}, y_{2,2}\right] x_{3}\left[y_{3,1}, y_{3,2}\right] x_{4}\left[y_{4,1}, y_{4,2}\right] x_{5} .
$$

With identical gluing, we have various identities, including

$$
\left\{x_{1},\left[y_{1,1}, y_{1,2}\right] x_{2}\left[y_{2,1}, y_{2,2}\right] x_{3}\left[y_{3,1}, y_{3,2}\right] x_{4}\left[y_{4,1}, y_{4,2}\right], x_{5}\right\}
$$

and

$$
x_{1}\left[y_{1,1}, y_{1,2}\right]\left\{x_{2},\left[y_{2,1}, y_{2,2}\right], x_{3}\right\}\left[y_{3,1}, y_{3,2}\right] x_{4}\left[y_{4,1}, y_{4,2}\right] x_{5} .
$$

Namely, we may take either of the transposed differences in which the nonzero substitutions that must be glued are interchanged.

The same argument yields the following result:

Proposition 8.3. If a path has identical gluing between the $i$ and $j$ components, with $i<j$, then each corresponding transposed difference taken from polynomials of Definition 4.11 yields an identity.

This gives us the general idea for defining $\Psi$ for paths with identical gluing.

Definition 8.4. Suppose we denote the glued indices of diagonal blocks as

$$
I_{j}:=\left\{i_{(j, 1)}, \ldots, i_{\left(j, t_{j}\right)}\right\},
$$

where $1 \leq j \leq \ell$. (In other words, we have $\ell$ sets of glued vertices.) For any index $i$, we write $\mu(i)$ for that $j$ such that $i \in I_{j}$. We define $\Psi(\Gamma)$ to be

$$
\sum_{\pi_{j} \in S_{t_{j}}} \operatorname{sgn} \pi_{1} \cdots \operatorname{sgn} \pi_{\ell} h_{\pi_{\mu(1)}} f_{1} h_{\pi_{\mu(2)}} f_{2} \cdots .
$$

For example, if vertices 1 and 4 have identical gluing and vertices 2 and 3 have identical gluing, then $\Psi(\Gamma)$ would be

$$
\begin{aligned}
& x_{1}\left[y_{11}, y_{12}\right] x_{2}\left[y_{21}, y_{22}\right] x_{3}\left[y_{31}, y_{32}\right] x_{4}\left[y_{41}, y_{42}\right] \\
- & x_{4}\left[y_{11}, y_{12}\right] x_{2}\left[y_{21}, y_{22}\right] x_{3}\left[y_{31}, y_{32}\right] x_{1}\left[y_{41}, y_{42}\right] \\
- & x_{1}\left[y_{11}, y_{12}\right] x_{3}\left[y_{21}, y_{22}\right] x_{2}\left[y_{31}, y_{32}\right] x_{4}\left[y_{41}, y_{42}\right] \\
+ & x_{4}\left[y_{11}, y_{12}\right] x_{3}\left[y_{21}, y_{22}\right] x_{2}\left[y_{31}, y_{32}\right] x_{1}\left[y_{41}, y_{42}\right] .
\end{aligned}
$$


Here is another identity arising from nonconsecutive, identical gluing along the diagonal, via the idea of Proposition 5.9.

Lemma 8.5. If there is a distance of $k$ between two diagonal blocks in the same glued component of a path of length $\ell$ in a full quiver whose algebra has radical of nilpotence index $\ell$, then the polynomial

$$
h_{n_{1}} f_{2} \cdots h_{n_{i-1}}\left[\ldots\left[\left[f_{i}, h_{i+1}\right], h_{i+2}\right], \ldots, h_{n_{i+k}}\right] h_{n_{i+k+1}} f_{i+k+2} \cdots h_{n_{\ell}}
$$

is an identity of the corresponding algebra, and is critical for $F$ infinite.

Proof. The evaluations of the $h_{i}$ must be in the correct semisimple block in order not to surpass the nilpotence index of the radical and yield 0 .

8.3. Weak Frobenius gluing above the diagonal. We arrive at the most problematic kind of gluing within a path: non-Frobenius gluing above the diagonal (which also requires diagonal gluing). Even for $F$ infinite, these quasi-linear relations yield new identities, by means of the Capelli polynomials $c_{n}\left(x_{1}, \ldots, x_{n} ; y_{1}, \ldots\right.$, $\left.y_{n}\right)$. [8, Example 5.3] is an extreme case, in which the underlying algebra is commutative. Here is a more subtle example.

Example 8.6. Consider

$$
\left\{\left(\begin{array}{ccccc}
\alpha & \beta & * & * & * \\
0 & \alpha & \beta+\gamma & * & * \\
0 & 0 & \alpha & \beta+\gamma & * \\
0 & 0 & 0 & \alpha & \beta \\
0 & 0 & 0 & 0 & \alpha
\end{array}\right): \alpha, \beta, \gamma \in F\right\} .
$$

The full quiver is

$$
I \stackrel{\beta}{\longrightarrow} I \stackrel{\beta+\gamma}{\longrightarrow} I \stackrel{\beta+\gamma}{\longrightarrow} I \stackrel{\gamma}{\longrightarrow} I .
$$

Then the Capelli polynomial $c_{3} \in \mathrm{id}(A)$, since the only nonzero evaluation must involve entries just above the diagonal, which are linearly dependent. One can get other identities involving commutators. For example, note that

$$
\begin{gathered}
\left.\left[\begin{array}{ccccc}
\alpha & \beta & * & * & * \\
0 & \alpha & \beta+\gamma & * & * \\
0 & 0 & \alpha & \beta+\gamma & * \\
0 & 0 & 0 & \alpha & \beta \\
0 & 0 & 0 & 0 & \alpha
\end{array}\right),\left(\begin{array}{ccccc}
\alpha^{\prime} & \beta^{\prime} & * & * & * \\
0 & \alpha^{\prime} & \beta^{\prime}+\gamma^{\prime} & * & * \\
0 & 0 & \alpha^{\prime} & \beta^{\prime}+\gamma^{\prime} & * \\
0 & 0 & 0 & \alpha^{\prime} & \beta^{\prime} \\
0 & 0 & 0 & 0 & \alpha^{\prime}
\end{array}\right)\right] \\
=\left(\begin{array}{ccccc}
0 & 0 & \beta \gamma^{\prime}-\gamma \beta^{\prime} & * & * \\
0 & 0 & 0 & 0 & * \\
0 & 0 & 0 & 0 & -\left(\beta \gamma^{\prime}-\gamma \beta^{\prime}\right) \\
0 & 0 & 0 & 0 & 0 \\
0 & 0 & 0 & 0 & 0
\end{array}\right),
\end{gathered}
$$

so $\left[\left[x_{1}, x_{2}\right],\left[x_{3}, x_{4}\right]\right]$ and $\left[\left[x_{1}, x_{2}\right], x_{5},\left[x_{3}, x_{4}\right]\right] \in \operatorname{id}(A)$. (The latter is because the only nonzero product comes from the $(1,3),(3,3)$, and $(3,5)$ positions.) 
In the presence of nonidentity Frobenius gluing on the diagonal, one must modify the Capelli polynomial to permit suitable $q$-powers of the indeterminates in certain monomials, analogously to Definition 4.24. Also, one should realize that changing the gluing coefficients in Example 8.6 might correspond to the same Capelli identities.

8.4. Interactions between branches. We turn briefly to the trickiest aspect of the subject, the interesting question as to how the polynomials of the branches can cancel and produce identities for the full quiver $\Gamma$. This is a very difficult problem, and we cannot give a full description of id $(\Gamma)$; rather we show how the full quivers naturally yield identities, via gluing between branches. In earlier sections, gluing was viewed as a nuisance, which eliminates useful trace-absorbing nonidentities and requires separate handling, but here we consider gluing in a positive light, yielding canonical new identities.

The different permuted branches $\mathcal{B}_{\pi}$ are obtained first by creating another branch with degenerate gluing and then permuting its edges. Thus, applying these permutations to the factors of the critical nonidentities of $\mathcal{B}$ yields evaluations that satisfy a given linear relation, and thus give rise to an identity.

For example, two permuted branches $\mathcal{B}_{1}$ and $\mathcal{B}_{2}$ have different polynomials $f_{1}=$ $\Phi\left(\mathcal{B}_{1}\right)$ and $f_{2}=\Phi\left(\mathcal{B}_{2}\right)$ whose evaluations must be proportional, and thus for suitable $\mu, f_{1}-\mu f_{2}$ is an identity of $A$.

In this way, permuted branches of a full quiver yield identities of the form

$$
\sum \mu_{k} \Phi\left(\mathcal{B}_{k}\right)
$$

These considerations give rise to the following example that counters intuition.

Example 8.7. Suppose the full quiver $\Gamma$ of the algebra $A$ is of the form

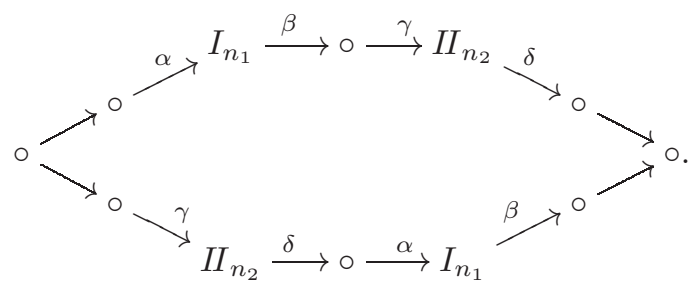

Here we take $n_{1} \neq n_{2}$, so that the corresponding central polynomials distinguish the blocks. There is one initial vertex and one terminal vertex. In the upper branch a block of type $I$ precedes a block of type $I I$, and the reverse is true in the lower branch.

Consider the polynomial

$$
P:=\Psi(\Gamma)=P_{1}-P_{2},
$$

where $P_{1}=f_{1} h_{n_{2}} f_{2} f_{3} h_{n_{4}} f_{4}$ and $P_{2}=f_{3} h_{n_{4}} f_{4} f_{1} h_{n_{2}} f_{2}$. The evaluations corresponding to each branch cancel, so $P=\left[f_{1} h_{n_{2}} f_{2}, f_{3} h_{n_{4}} f_{4}\right]$ vanishes identically on A.

Note that $A$ is a factor algebra of $A_{1} \oplus A_{2}$, where $A_{1}$ and $A_{2}$ are the algebras corresponding to the two branches. Thus, letting $\mathcal{F}$ denote the free algebra, we see that $\operatorname{id}(A)=\operatorname{id}\left(A_{1}\right) \cap \operatorname{id}\left(A_{2}\right)$, and thus the relatively free algebra $\hat{A}=\mathcal{F} / \operatorname{id}(A)$ of $A$ is a subdirect product of $\mathcal{F} / \operatorname{id}\left(A_{1}\right)$ and $\mathcal{F} / \operatorname{id}\left(A_{2}\right)$. 
On the other hand, the natural morphism

$$
\hat{A} \quad \rightarrow \quad \mathcal{F} /\left(\langle P\rangle+\operatorname{id}\left(A_{1}\right)\right) \oplus \mathcal{F} /\left(\langle P\rangle+\operatorname{id}\left(A_{2}\right)\right)
$$

is not monic since $\langle P\rangle+\mathrm{id}\left(A_{1}\right)$ contains $P_{1}$ and thus contains $P_{2}=P-P_{1}$ (and likewise $\langle P\rangle+\operatorname{id}\left(A_{2}\right)$ contains $P_{2}$ and $P_{1}=P-P_{2}$, whereas id $(A)$ contains neither $P_{1}$ nor $\left.P_{2}\right)$.

\section{REFERENCES}

[1] Belov, A., Counterexamples to the Specht problem, Sb. Math. 191, 329-340, (2000). MR.1773251 (2001g:16043)

[2] Belov, A., Local finite basis property and local representability of varieties of associative rings, Izvestia of Russian Academia of Science, 1, 3-134, (2010). English transl.: Izvestiya: Mathematics, 74(1), 1-126, (2010). MR2655238(2011e:16039)

[3] Belov, A., On varieties generated by a ring which is finite-dimensional over its centroid, Comm. Moscow Math. Soc., Russian Math. Surveys 62:2, pp. 379-400. MR2352369 (2008e:16019)

[4] Belov, A., The Gel'fand-Kirillov dimension of relatively free associative algebras, Sbornik Math. 195, 1703-1726, (2004). MR2138478(2006c:16041)

[5] Bernšteĭn, I. N., Gel'fand, I. M. and Ponomarev, V. A. Coxeter functors, and Gabriel's theorem (Russian), Uspehi Mat. Nauk 28(2(170)), 19-33, (1973). MR0393065 (52:13876)

[6] Belov, A. and Rowen, L.H. Computational Aspects of Polynomial Identities, Research Notes in Mathematics 9, AK Peters, 2005. MR2124127 (2006b:16001)

[7] Belov, A., Rowen, L.H., and Vishne, U., Structure of Zariski-closed algebras, Trans. Amer. Math. Soc., 362, 4695-4734, (2010). MR2645047

[8] Belov, A., Rowen, L.H., and Vishne, U., Full quivers of representations of algebras, Trans. Amer. Math. Soc., to appear.

[9] Belov, A., Rowen, L.H., and Vishne, U., Application of full quivers to polynomial identities, Comm. in Alg., to appear (Jan. 2012).

[10] Belov, A., Rowen, L.H., and Vishne, U., Specht's problem for affine algebras over arbitrary commutative Noetherian rings, preprint.

[11] Drensky, V. S. Identities in Lie algebras. (Russian) Algebra i Logika 13 (1974), 265-290, 363-364. MR0374220 (51:10420)

[12] Giambruno, A. and Zaicev, M., Minimal varieties of algebras of exponential growth, Adv. Math. 174 (2003), 310-323. MR1963697 (2004b:16028)

[13] Giambruno, A. and Zaicev, M., "Polynomial identities and asymptotic methods", Math. Surveys and Monographs, vol. 122, Amer. Math. Soc., 2005. MR2176105 (2006g:16054)

[14] Grishin,A.V., Examples of T-spaces and T-ideals in Characteristic 2 without the Finite Basis Property (in Russian), Fundam. Prikl. Mat. 5 (1), no. 6, 101-118 (1999). MR.1799541 (2002a:16028)

[15] Jacobson, N., Basic Algebra II, Freeman (1980). MR.571884 (81g:00001)

[16] Kemer, A.R., The representability of reduced-free algebras, Algebra i Logika 27(3), 274-294, (1988). MR997959(90e:16027)

[17] Kemer, A.R., Identities of finitely generated algebras over an infinite field, Math. USSR Izv. 37, 69-97, (1991). MR1073084 (91j:16027)

[18] Kemer, A.R., Identities of Associative Algebras, Transl. Math. Monogr., 87, Amer. Math. Soc. (1991). MR1159223(93d:16027)

[19] Kambayashi, Tatsuji; Miyanishi, Masayoshi; Takeuchi, Mitsuhiro. Unipotent algebraic groups, Lecture Notes in Mathematics 414, Springer-Verlag, Berlin-New York, 1974. MR0376696 (51:12871)

[20] Lewin, J., A matrix representation for associative algebras. I and II, Trans. Amer. Math. Soc. 188(2), 293-317 (1974). MR0338081(49:2848)

[21] Oates, S. and Powell, M.B., Identical relations in finite groups, J. Algebra 1(21), 11-39, (1964). MR0161904(28:5108) 
[22] Rowen, L.H., Polynomial identities in ring theory, Pure and Applied Mathematics 84, Academic Press (1980). MR.576061 (82a:16021)

[23] Rowen, L.H., Ring theory, Vol. 1, Pure and Applied Mathematics 127, Academic Press (1988). MR940245(89h:16001)

Department of Mathematics, Bar-Ilan University, Ramat-Gan 52900, Israel

E-mail address: belova@macs.biu.ac.il

Department of Mathematics, Bar-Ilan University, Ramat-Gan 52900, Israel

E-mail address: rowen@macs.biu.ac.il

Department of Mathematics, Bar-Ilan University, Ramat-Gan 52900, Israel

E-mail address: vishne@macs.biu.ac.il 